\title{
Modulations of the processing of line discontinuities under selective attention conditions?
}

\author{
ANNE GIERSCH \\ Hôpitaux Universitaires de Strasbourg, Strasbourg, France \\ and \\ MANFRED FAHLE \\ University of Bremen, Bremen, Germany
}

\begin{abstract}
We examined whether the processing of discontinuities involved in figure-ground segmentation, like line ends, can be modulated under selective attention conditions. Subjects decided whether a gap in collinear or parallel lines was located to the right or left. Two stimuli were displayed in immediate succession. When the gaps were on the same side, reaction times (RTs) for the second stimulus increased when collinear lines followed parallel lines, or the reverse, but only when the two stimuli shared the same orientation and location. The effect did not depend on the global form of the stimuli or on the relative orientation of the gaps. A frame drawn around collinear elements affected the results, suggesting a crucial role of the "amodal" orthogonal lines produced when line ends are aligned. Including several gaps in the first stimulus also eliminated RT variations. By contrast, RT variations remained stable across several experimental blocks and were significant for interstimulus intervals from 50 to $600 \mathrm{msec}$ between the two stimuli. These results are interpreted in terms of a modulation of the processing of line ends or the production of amodal lines, arising when attention is selectively drawn to a gap.
\end{abstract}

The present study is aimed at exploring whether the processing of line discontinuities involved in figure-ground segmentation, like line ends and gaps, can be modulated, especially when attention is selectively drawn to one part of an object.

Objects in everyday life can be perceived and analyzed in a general or a more detailed way. Depending on requirements, it is possible to focus on one part of a complex object or inspect an object as a whole. It is highly probable that top-down processes and attentional control modulate the balance between the perception of whole objects and the perception of object parts (Chey, Grossberg, \& Mingolla, 1997; Humphreys \& Riddoch, 1994). For instance, Humphreys and Riddoch proposed that attentional processes control the coding of the relations both between objects and within objects. Chey et al. (1997) simulated the integration of local motions into a global coherent motion. Their model contains an attentional feedback originating from the cortical area MST, with excitatory inputs onto all MT cells coding for a primed direction and inhibitory inputs onto all other direction-sensitive cells in area MT.

Focusing on a part of an object may also imply the modulation of more primitive signals. The question we address is whether focusing on whole objects as opposed to parts

This research was funded by a grant from INSERM. Correspondence should be addressed to A. Giersch, Hôpitaux Universitaires de Strasbourg, INSERM U405, Département de Psychiatrie I, 1, pl de l'Hôpital, 67091 Strasbourg Cedex, France (e-mail: giersch@alsace.u-strasbg.fr). of objects also involves the modulation of the coding of signals processed at an earlier level, like discontinuities in lines. To this end, we will begin by checking whether the coding of discontinuities can indeed be modulated. Line ends, borders, and discontinuities are considered as primitives and coded as early as V1 and V2 (DeAngelis, Freeman, \& Ohzawa, 1994; Duysens, Orban, van der Glas, \& de Zegher, 1982; Duysens, Orban, van der Glas, \& Maes, 1982;Heitger, Rosenthaler, von der Heydt, Peterhans, \& Kubler, 1992; Hubel \& Wiesel, 1965; Orban, Kato, $\&$ Bishop, 1979a, 1979b). These signals, together with other primitives like orientation or spatial frequency, are input signals for integration and segmentation processesthat is, the binding of local features. The content of these signals can influence the way information is bound together. For instance, the luminance of line ends is a physical property that influences whether forms are perceived as integrated entities or separate parts (Lorenceau \& Shiffrar, 1992; Shiffrar \& Lorenceau, 1996).

Several results in the literature suggest that the processing of primitives like discontinuities in lines may be modulated. Both psychophysical and electrophysiological studies indicate that the coding of image primitives is more plastic than once believed. For instance, studies exploring hyperacuity phenomena show that performance improves with time when subjects learn to detect offsets below the diameter of foveal photoreceptors between parallel line segments (Fahle, 1994; McKee \& Westheimer, 1978). This learning effect is highly orientation and position depen- 
dent and partly monocular (Poggio, Fahle, \& Edelman, 1992). Performance returns to base level when the orientation or position of stimuli is changed and also at least partly when stimuli are presented to the other eye than that tested in the training phase. These characteristics indicate that learning takes place at least partly at an early level of processing (Fahle, 1996). Electrophysiologicalstudies also indicate that neurones in the primary visual cortex can modify their response pattern as a result of nearby lesions or as a function of the visual information presented outside their receptive fields (Bringuier, Chavane, Glaeser, \& Frégnac, 1999; Eysel, Eyding, \& Schweigert, 1998; Gilbert, Das, Ito, Kapadia, \& Westheimer, 1996; Kapadia, Ito, Gilbert, \& Westheimer, 1995; McLean \& Palmer, 1998). Anatomical and electrophysiological studies show that there are huge feedback connections from higher order areas to lower order areas such as V1 and V2 (Kennedy \& Bullier, 1985; Salin \& Bullier, 1995; Shipp \& Zeki, 1989). Hupé et al. (1998) showed, for example, that inactivating area V5 decreases the responses to a moving central bar of low salience in areas V1, V2, and V3, and increases the responses to a bar when it moves coherently with the background. These results suggest that feedback connections from area $\mathrm{V} 5$ have a facilitatory effect on discrimination between figure and ground in areas V1, V2, and V3. There is converging evidence for top-down influences on the early processing of visual information. However, to our knowledge, the functional role of the top-down influences on the processing of discontinuities in lines has not been studied in the field of experimental psychology.

Flexibility in the processing of discontinuities in lines might be useful in several ways. Each discontinuity is inherently ambiguous. It can either represent a true line end, or it can result from an occlusion (Biederman, 1987; Lorenceau \& Shiffrar, 1992; Shimojo \& Nakayama, 1990; Shimojo, Silverman, \& Nakayama, 1989). Even a simple stimulus composed of two collinear line segments can be interpreted as two separate parts or as one single line with an interruption. As noted, such an interpretation probably occurs at a late stage of the processing of visual information, but early processes might also play a role. Indeed, electrophysiological studies show that equivalent responses for solid lines and lines made of aligned dots can be observed as early as cortical area V2 in monkeys (Peterhans \& von der Heydt, 1991), and perhaps V1 (Grosof, Shapley, \& Hawken, 1993; Redies, Crook, \& Creutzfeldt, 1986; Sheth, Sharma, Rao, \& Sur, 1996). Such an early completion of partly occluded contours might be useful when the line ends result from an occlusion (Giersch, Humphreys, Boucart, \& Kovacs, 2000; Rensink \& Enns, 1998; Sekuler $\&$ Palmer, 1992). We will call these types of amodal contour "Type 1." Such contours are inconsistent with the presence of a true gap between two separated line segments. Some studies suggest indeed that completion hinders the detection of gaps. Nakayama, Shimojo, and Silverman (1989; see also Shimojo \& Nakayama, 1990; Shimojo et al., 1989) proposed that borders defined by occlusion are removed from further analysis so that they help the group- ing with other borders defined by occlusion. Using a visual search task, Rensink and Enns (1998) showed that the presence of an occluder prevented subjects from using the gap between the two visible collinear parts of the occluded object. Furthermore, it has been shown recently that H.J.A., an agnosic patient impaired at binding contours to shapes, was nevertheless able to complete missing gaps (Giersch et al., 2000), at least when the occluded contour was sufficiently short. However, when he was able to complete the gap, he found it more difficult to discriminate occluded from visible contours. He behaved as if the gap produced by occlusion was completed by a real line. Hence, detecting a gap between two collinear line segments may involve the modulation of two types of signals. It might be useful to enhance the processing of the line ends signaling the gap and to inhibit the production of an amodal line linking the two line segments, these two mechanisms being complementary. In contrast, if the gap is composed of two parallel line segments (Figure 1a, lower panel), completion of the gap by interpolation should not occur, because the two line segments are not "relatable" (Kellman \& Shipley, 1991). However, such a stimulus includes two aligned line ends and aligned line ends are known to produce virtual connecting lines (Grossberg \& Mingolla, 1985; Lesher \& Mingolla, 1993; Shipley \& Kellman, 1990; Westheimer \& Li, 1996; Zucker \& Davis, 1988). These virtual contours may be produced by mechanisms different from those underlying the completion of occluded contours. We will call these types of contour "Type 2." The presence of line ends is crucial in the case of orthogonal virtual lines (Lesher, 1995), whereas they are somewhat detrimental for amodal contours bridging gaps ("Type 1" contours; Nakayama et al., 1989; Shimojo et al., 1989). However, as for amodal lines linking collinear contours, virtual lines orthogonal to line ends can be recorded as early as V2 (von der Heydt $\&$ Peterhans, 1989). According to von der Heydt and Peterhans, responses can be recorded in the presence of only two line ends. The quantitative aspects of the results observed at an electrophysiologicallevel by von der Heydt and Peterhans were confirmed recently at a psychophysical level by Gurnsey, Iordanova, and Grinberg (1999). Hence, even if such signals are weak when only two line ends are present and do not give rise to any conscious perception, they may exist and influence the processing of visual information. ${ }^{1}$

In the case of collinear line segments, gaps are defined by two line ends. Amodal orthogonal contours (Type 2) might thus be produced at the end of the lines composing the gap (Figure 1b), but they would not link the line ends and would be consistent with the presence of a gap. In contrast, if the gap is located between two aligned line ends (the line segments being parallel), orthogonal contours (still Type 2) would link the line ends and would be inconsistent with an interpretation in terms of a true gap between the two lines (Figure 1c). Since the line-end signals play an important role in the production of virtual orthogonal lines (Type 2), increasing the strength of the line-end signals would impair the detection of a gap be- 
a
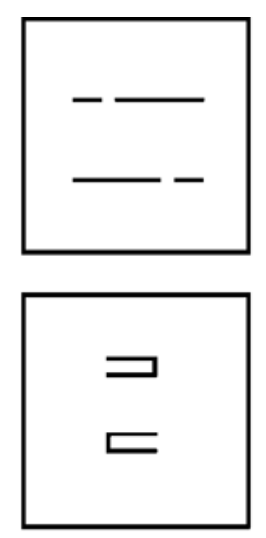

b

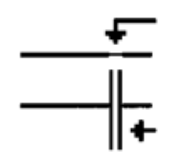

c

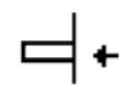

d

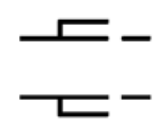

\section{$\mathbf{e}$}

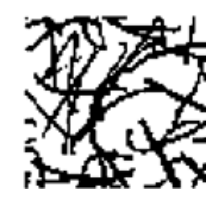

Figure 1. (a) Illustration of the stimuli used in Experiment 1, either collinear (upper panel) or parallel (lower panel). The stimuli were presented one at a time. (b) Illu stration of the "amodal"lines produced by stimuli composed of collinear elements, and (c) of parallel elements. (d) The stimuli are drawn superimposed to illustrate the fact that the stimuli were presented so that the discontinuities on one side were always in the same location on the screen. (e) Illustration of the mask displayed between each sequence of two stimuli.

tween two parallel line segments, in contrast to a gap between two collinear line segments. In the case of parallel line segments, it might thus be useful to reduce the strength of these orthogonal Type 2 contours, either directly or by reducing instead of increasing the strength of the line-end signals producing these contours. If the processing of such signals is indeed modulated, the modulations should vary as a function of the configuration of the gap, and the visual system might "switch on" the appropriate mechanisms. It follows that the perception of a gap in a given configuration might affect the perception of a gap in another configuration. For example, when the task is to detect a gap between two collinear line segments, detection for line-end signals might be activated. This activation might in turn be deleterious when the task is to detect a gap between two aligned, parallel line ends. Hence, we tested the hypothesis that the processing of discontinuities is modulated by testing how a gap in a given configuration affects the detection of a gap in another configuration. We chose these two configurations, collinear and parallel line segments, for the following reasons: (1) The ambiguity of the stimuli made it possible to test our hypotheses. (2) Modulations of the processing of discontinuities in healthy volunteers treated with benzodiazepines were already observed in previous studies with similar stimuli (Giersch, 1999). (3) Finally, we assumed that the two types of gaps were not incompatible a priori and that attentional or decisional effects involved in the processing of one type of gap would not be deleterious for the processing of the other type of gap. However, it should be noted that no direct inference concerning the level at which modulations occur can be made from the results at this stage, even if our predictions are met. These aspects will be discussed further in the following experiments.

We used a modified short-term priming task to examine whether performance varies in healthy volunteers as a function of the prior exposure to a stimulus. Stimuli were composed of horizontal line segments, which were either collinear or parallel. Line segments were separated by a gap located either to the right or to the left (Figure 1). This gap between the line ends was horizontal in the case of collinear line segments and vertical in the case of parallel line segments. Subjects were instructed to decide whether the gap was located to the left or the right. If presentation of a first stimulus primes the location of the gap irrespective of its physical properties, performance should be better when the gap remains on the same side in the two consecutive stimuli, whatever the arrangement of line segments and the orientation of the gap. However, if the detection of a gap depends more on its physical properties, the advantage for the second gap on the same side may be lost or even reversed when the two consecutive stimuli differ in terms of certain physical properties.

\section{EXPERIMENT 1}

\section{Method}

Subjects. Subjects were 8 paid students attending the University of Tübingen. They had normal or corrected-to-normal visual acuity and were naive as to the aim of the study.

Apparatus. The stimuli were displayed on a color raster monitor. They were generated using a microcomputer equipped with a SVGA graphic card. Screen resolution was $640 \times 480$ pixels. Each pixel sub- 
tended $2.4^{\prime}$ of arc horizontally and vertically. Stimuli were presented in gray on a black background. In a dimly illuminated room the luminance of the stimuli was $8 \mathrm{~cd} / \mathrm{m}^{2}$ and the luminance of the background was $0.04 \mathrm{~cd} / \mathrm{m}^{2}$. The viewing distance of $57 \mathrm{~cm}$ was kept constant using a chinrest with a forehead support.

Stimuli. Stimuli were composed of horizontal line segments. The width of the lines was 1 pixel (2.4' of arc). Stimuli with a horizontal gap were composed of two collinear line segments: a 7-pixel-long line segment (16.8' of arc) and a 21-pixel-long line segment (50.4' of arc), separated by a gap of 3 pixels ( $7.2^{\prime}$ of arc). The gap was located either to the right or to the left side. Stimuli with a vertical gap were composed of two 12-pixel-long parallel line segments (28.8' of arc) separated by a gap of 3 pixels ( $7.2^{\prime}$ of arc). A vertical line linked the ends of the two line segments on one side, leaving a gap at the other end (Figure 1). Stimuli were arranged so that the gaps on one side were all in the same location on the screen, whatever the type of stimuli (Figure 1d). The stimulus composed of parallel elements was displayed in the exact center of the screen. The stimulus composed of collinear lines was displayed in equal proportion to the location of the uppermost and lowest line segments composing the "parallel" stimulus.

Procedure. A first stimulus was displayed in the center of the screen. Subjects were instructed to decide whether there was a gap to the right or the left of the stimulus and to press a right or left keyboard button accordingly. The stimulus stayed on the screen until the subjects responded, and then disappeared (the screen went black). A second stimulus followed after a delay of $100 \mathrm{msec}$. Subjects were instructed to decide again on which side the gap was located, and the stimulus disappeared after they gave their responses. Following the display of a mask for $100 \mathrm{msec}$ (Figure 1e) and a 1,000-msec interval during which the screen remained black, the sequence started again. The gaps appearing in the first and second stimuli in each sequence were either horizontal or vertical, resulting in four possible conditions: (1) The gap was horizontal in both the first and the second stimuli (composed of collinear line segments). (2) The gap was horizontal in the first stimulus (composed of collinear line segments) and vertical in the second one (composed of parallel line segments). (3) Both gaps were vertical (both stimuli were composed of parallel line segments). (4) The gap was vertical in the first stimulus (composed of parallel line segments) and horizontal in the second one (composed of collinear line segments). In each condition, the gap was either on the same side or on opposite sides in the two consecutive stimuli, defining a total of eight experimental conditions. The characteristics of the trials were randomly and equally represented: the side of the gap in the first and in the second stimuli, the position of the horizontal collinear elements (see Figure 1c), and the eight experimental conditions. The onset of the stimulus activated the computer clock, which was stopped when the subject pressed a key. Errors were signaled by a 300-msec sound, initiated after each incorrect response. These trials were not replaced, and these RTs were not taken into account in the following analysis. When there was an error for the first stimulus of a sequence, RTs observed for the second stimulus were also excluded from the analysis. The same procedure was applied in all following experiments.

\section{Results}

Analyses of variance (ANOVAs) were conducted on reaction times (RTs) and errors, with subjects as the random variable. There were four within-subjects variables: first stimulus type (with a horizontal or a vertical gap - that is, with collinear or parallel line segments), gap orientation (identical in the first and second stimuli vs. different), gap location (on the same vs. opposite sides in the first and second stimuli), and time of presentation (first vs. second of a pair). The tasks were very easy and errors did not show any significant effect or any speed-accuracy tradeoff. Whatever the experiment, errors were always lower than $2 \%$. We will thus describe only the results observed for RTs (displayed in Figure 2).

RTs to the first stimulus were equivalent whether the gap was horizontal (535 msec, line segments being collinear) or vertical (531 msec, line segments being parallel) $[F(1,7)=2.8, p>.13]$. RTs decreased between the first and second stimulus by $34 \mathrm{msec}[F(1,7)=7.7, p<.05]$. This decrease was not homogeneous across conditions and resulted in varying performances for the second stimulus. When the orientation of the gap was identical in the two consecutive stimuli (both horizontal or both vertical), RTs were shorter by $39 \mathrm{msec}$ when the gap was on the same side in both stimuli than when it was on opposite sides $[F(1,7)=6, p<.05]$. In contrast, when the two stimuli differed in terms of the orientation of their gap, RTs were higher by $59 \mathrm{msec}$ when the gap was on the same side in both stimuli than when it was on opposite sides $[F(1,7)=$ $16.6, p<.005$ (Figure 2)]. These effects resulted in a significant interaction between the relative orientation of the gap in the two stimuli (identical vs. different), gap location (on the same vs. opposite sides in the two stimuli), and time of presentation (first vs. second of a pair) $[F(1,7)=17.8$, $p<.005]$.

\section{Discussion}

When gaps appearing in the first and second stimuli were both horizontal or both vertical (both stimuli composed of collinear or parallel elements), subjects were faster when the gap was on the same side in both stimuli than when it was on opposite sides. In contrast, when the gaps in the first and second stimuli were orthogonal (one of the stimuli composed of collinear line segments and the other one of parallel line segments), subjects were slower when both gaps were on the same side than when they were on opposite sides. These results show that, in the present paradigm, the side of the gap is not primed independently from the physical properties of the stimuli. At this stage, however, there could be several explanations for the RT disadvantage observed when the gaps of the two consecutive stimuli are orthogonal and on the same side. In particular, the disadvantage might be attributable to the change in the global form of the two consecutive stimuli. This difference might be detected first and bias subjects to shift their response to the other side. Subjects would perform faster when the side of the gap is indeed shifted between the first and second stimuli than when it remains on the same side. A second possibility is that the detection of a gap in a given orientation impairs the detection of a gap whose orientation is orthogonal. Finally, the impairment might be the consequence of the collinear and parallel arrangement of the line segments. These hypotheses were explored further in Experiments 2, 3, and 4 by testing the effect of the global form of the stimuli (Experiment 2), the orientation of the gap (Experiment 3), and the orientation selectivity and the location specificity (Experiment 4) of the effects observed in Experiment 1. 


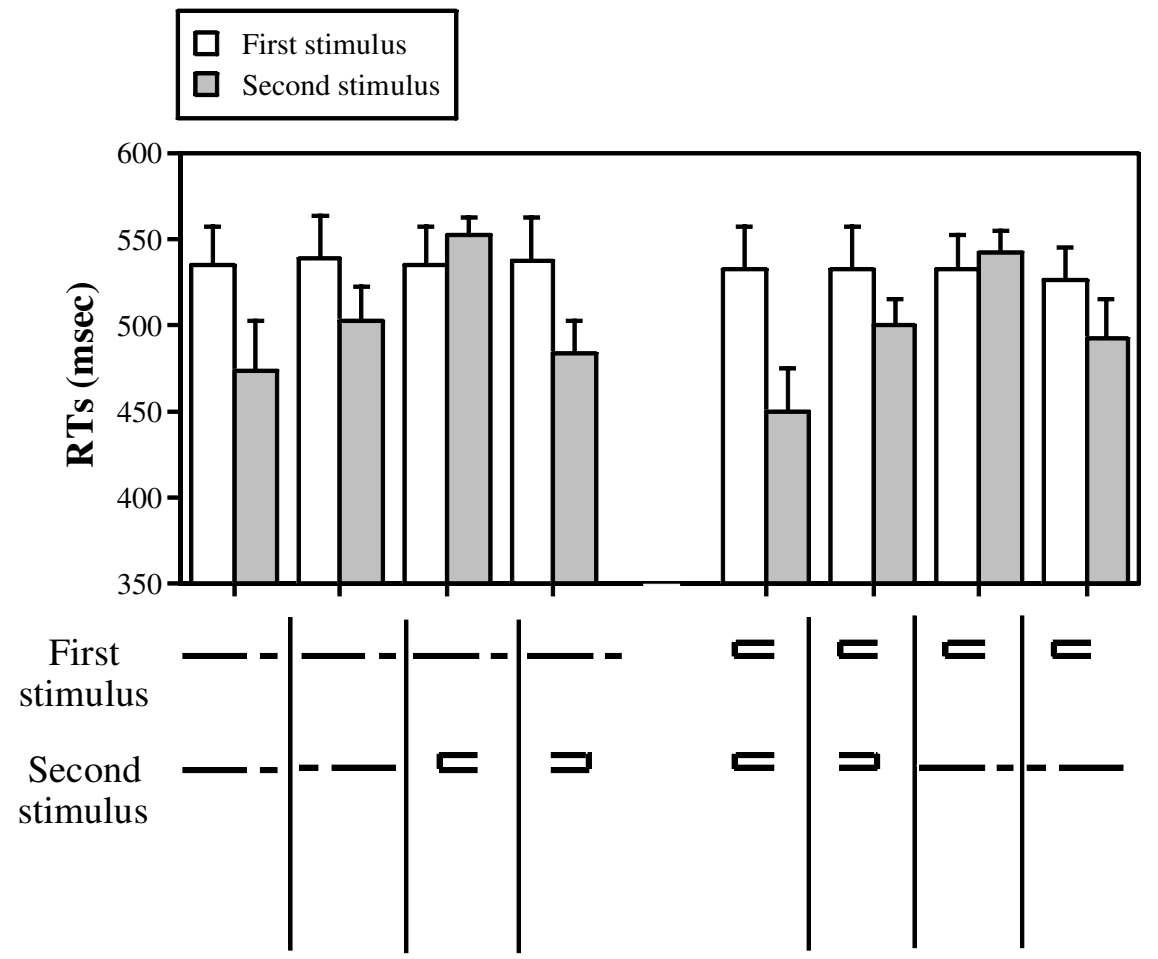

Figure 2. Mean reaction times (RTs) in Experiment 1 averaged across the 8 subjects (with standard errors) for the first stimulus (columns in white) and for the second stimulus (columns in black), as a function of experimental condition. Experimental conditions are defined by the collinear or parallel type of the first stimulus (upper drawing), the type of second stimulus (lower drawing), and the relative side of the discontinuities (on the same side vs. on opposite sides). The proportions of right and left gaps are identical in all conditions.

\section{EXPERIMENT 2}

In Experiment 2, we tested whether the slowing down observed when the two consecutive stimuli have a gap on the same side depends on the difference in the global form of the stimuli with collinear and parallel elements. To this end, we modified the stimuli composed of collinear and parallel elements so that their global form became very similar. If the change in global form is responsible for the slowing down observed when the two consecutive gaps are on the same side, then this effect should at least decrease when the two types of stimuli are more similar. In contrast, if the effect is independent from the change in global form between the two consecutive stimuli, the effect should be preserved even if the stimuli have the same global form.

\section{Method}

Subjects. Subjects were 8 new students attending the University of Strasbourg. They had normal or corrected-to-normal visual acuity and were naive as to the aim of the study.

The procedure and apparatus were identical to those used in Experiment 1 . There were two experimental blocks. The first block was identical to Experiment 1. The second block included stimuli that were similar in terms of their global form. The order of the two experimental blocks was randomized across subjects.
Stimuli. In the second experimental block (stimuli of similar global form), stimuli composed of horizontal collinear line segments composed rectangles with 15-pixel-long line segments ( $36^{\prime}$ of arc), and 5 -pixel-long vertical line segments $\left(12^{\prime}\right.$ of arc). The stimulus included a horizontal gap of 3 pixels ( $7.2^{\prime}$ of arc), always found in the uppermost line segment, and separating a 3-pixel-long line segment (7.2' of arc) from a 9-pixel-long line segment $\left(21.6^{\prime}\right.$ of arc; lower panel of Figure 3). The gap was located to either the right or the left, so that there were two possible locations for the gap, as in Experiment 1 and as with the stimulus composed of parallel line segments. Stimuli with a vertical gap were identical to those used in Experiment 1. Stimuli were still arranged so that the gaps on one side were all at the same location on the screen, whatever the type of stimuli.

\section{Results}

Stimuli differing in their global form. The results in this experimental block, displayed in the upper panel of Figure 3, reproduced those observed in Experiment 1. RTs were equivalent for stimuli composed of collinear elements (407 msec) and parallel elements (408 msec).

RTs tended to decrease by 19 msec between the first and second stimuli $[F(1,7)=4.5, p=.072]$. This decrease was not homogeneous across conditions, as suggested by a significantinteraction between the relative arrangement of the two stimuli (identical vs. different), the gap location (on the same vs. opposite sides in the two stimuli), and the 

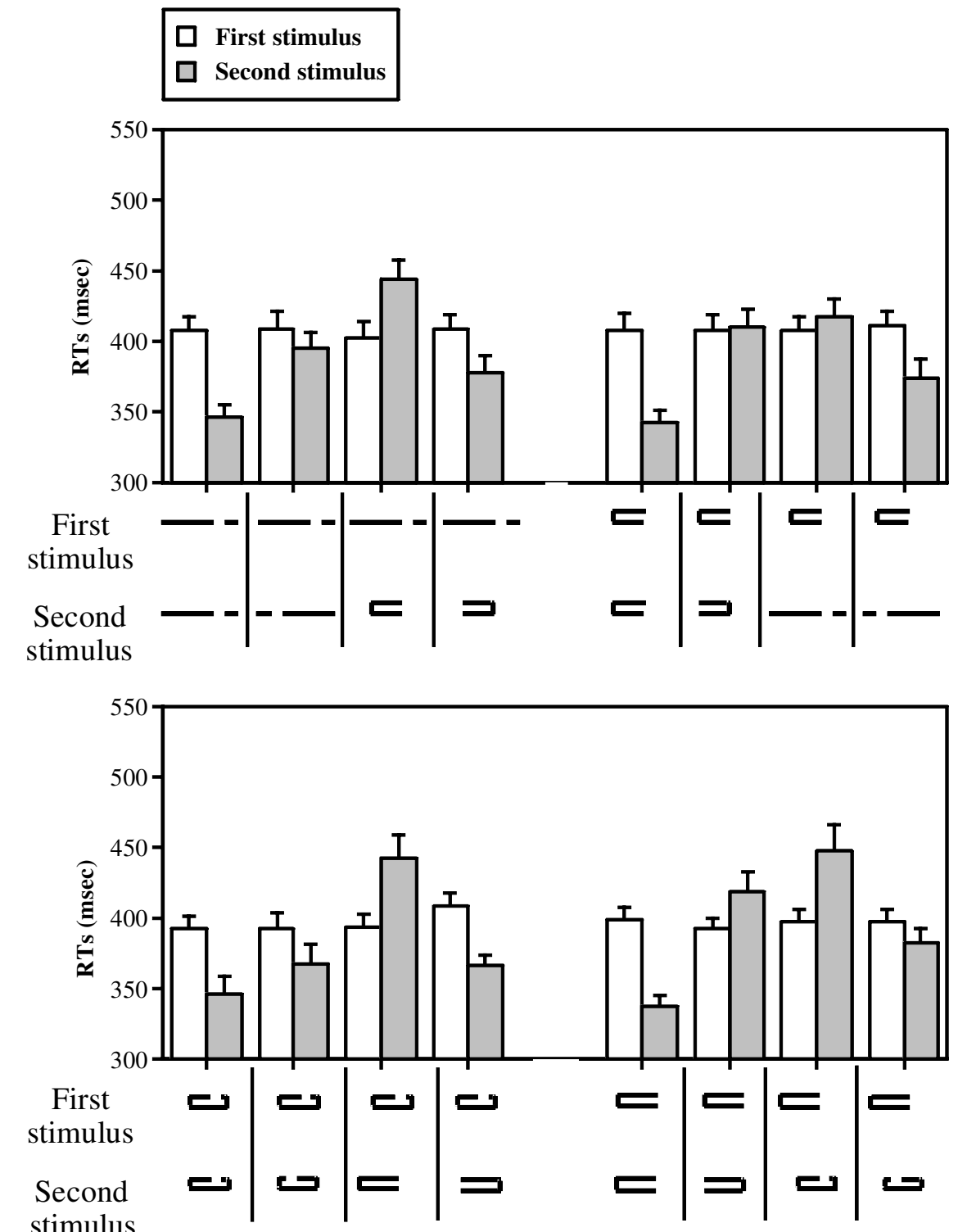

Figure 3. Mean reaction times (RTs) (with standard errors) in Experiment 2 averaged across the 8 subjects for the first stimulus (columns in white) and for the second stimulus (columns in black), as a function of experimental condition, when the stimuli are similar in their global form (lower panel) and when they are not (upper panel).

time of presentation (first vs. second of a pair) $[F(1,7)=$ $75.7, p<.001]$. When the orientation of the gap was identical in the first and in the second stimuli (both horizontal or both vertical), RTs to the second stimulus were shorter by $58 \mathrm{msec}$ when the gap was on the same side in the two consecutive stimuli than when it was on opposite sides $[F(1,7)=18.4, p<.005]$. In contrast, when the first and the second stimulus differed in terms of the orientation of their gaps, RTs to the second stimulus were higher by $53 \mathrm{msec}$ when the gap was on the same side than on opposite sides in the two consecutive stimuli $[F(1,7)=6.9, p<.05]$.
Stimuli similar in terms of their global form. RTs to the first stimulus were similar for stimuli made up of collinear line segments (397 msec) and for stimuli made up of parallel line segments $(396 \mathrm{msec})(F<1)$. RTs decreased only by $8 \mathrm{msec}$ between the first and the second stimuli $[F(1,7)=22.1, p<.005]$. This decrease was not homogeneous across conditions. When the orientation of the gap was identical in the two consecutive stimuli (both horizontal or both vertical), RTs to the second stimulus were shorter by $52 \mathrm{msec}$ when the gap was on the same side in the two consecutive stimuli than when it was on opposite sides 
$[F(1,7)=16.9, p<.005]$. When the two consecutive stimuli differed in terms of the orientation of their gap, RTs to the second stimulus were higher by $71 \mathrm{msec}$ when the gap was on the same side in both stimuli than when it was on opposite sides $[F(1,7)=16.3, p<.005]$. These effects resulted in a significant interaction between the relative orientation of the gap in the two stimuli (identical vs. different), gap location (on the same vs. opposite sides in the two stimuli), and time of presentation (first vs. second of a pair) $[F(1,7)=104.2, p<.001]$.

Comparison between the two experimental blocks. RTs to the first stimulus did not vary significantly across experimental blocks $\left(F_{\mathrm{S}}<1\right)$. When the orientation of the gap differed in the two consecutive stimuli (one vertical and one horizontal), the RT disadvantage observed when the two consecutive gaps were on the same side was slightly but significantly larger, by $18 \mathrm{msec}$, for stimuli of similar global form than for stimuli of different global form $[F(1,7)=16.1, p<.005]$. This resulted in a significant interaction between the experimental blocks (with stimuli of similar or different global form), the relative arrangement of the first and second stimuli (identical vs. different in terms of the orientation of the gap), gap location (on the same vs. opposite sides in the two consecutive stimuli), and time of presentation (first vs. second of a pair) $[F(1,7)=5.8, p<.05]$.

\section{Discussion}

As in Experiment 1, when the gaps of the two consecutive stimuli were orthogonal (one stimulus with a gap between collinear elements and one stimulus with a gap between parallel elements), subjects were slower when the two consecutive gaps were on the same side than when the gaps were on opposite sides. When stimuli were similar in terms of their global form, the effect failed not only to decrease, but actually increased slightly. These results show that the change in the global form between the two consecutive stimuli is not responsible for the RT variations observed when the gaps are on the same or on opposite sides. The change in the global form of the two consecutive stimuli may prompt subjects to adjust the mechanisms used to process discontinuities.

\section{EXPERIMENT 3}

In Experiment 3, we checked whether RT variations were due to the orthogonality of gaps. Stimuli were distorted so that gaps were superimposed and not orthogonal anymore when stimuli differed regarding the arrangement of their global form (composed of collinear or parallel elements). Stimuli consisting of parallel line segments were distorted so that the amodal line (Type 2) linking the two line ends of this stimulus formed an acute angle with the line ends (lower panel of Figure 4). For stimuli consisting of collinear line segments, the shorter line segment of the collinear stimulus was shifted upward or downward so that the stimulus was not collinear anymore. In both cases, the gaps became oblique.

\section{Method}

Subjects. Subjects were 8 new students attending the University of Strasbourg. They had normal or corrected-to-normal visual acuity and were naive as to the aim of the study.

The procedure and apparatus were identical to those used in Experiment 1 . There were two experimental blocks. The first block was identical to that in Experiment 1. The second block included stimuli with oblique gaps. The order of the two experimental blocks was randomized across subjects.

Stimuli. In the second experimental block, the shorter line segment of the collinear stimulus was shifted upward or downward by 3 pixels $\left(7.2^{\prime}\right.$ of arc), so that the two line segments were at the same height as in the parallel stimulus. In regard to the stimulus consisting of parallel elements, one of the two horizontal line segments was shifted toward the outer limit of the screen (by 5 pixels, i.e., 12' of arc) and was connected with the other line segment by an oblique line segment. Stimuli were displayed on the screen so that gaps on the same side of the stimuli were always exactly superimposed.

\section{Results}

Orthogonal gaps. The results, displayed in the upper panel of Figure 4, were identical to those described in the preceding experiments. RTs for the first stimuli are not detailed in the graph, since they are perfectly homogeneous across conditions; they are displayed in the following experiments only when there is some effect of the configuration of the first stimuli. RTs to the first stimulus were equivalent for stimuli composed of collinear elements $(418 \mathrm{msec})$ and for stimuli composed of parallel elements $(415 \mathrm{msec})(F<1)$. RTs decreased only by $8 \mathrm{msec}$ between the first and the second stimuli $(F<1)$. Once again, this decrease was not homogeneous across conditions, as suggested by a significant interaction between the relative arrangement of the two stimuli (identical vs. different), gap location (on the same vs. opposite sides in the two stimuli), and time of presentation (first vs. second of a pair) $[F(1,7)=33.4, p<.001]$. When the two consecutive stimuli shared the same arrangement of line segments (both collinear or both parallel), RTs to the second stimulus were shorter by $49 \mathrm{msec}$ when the gap was on the same side in the two stimuli than when on opposite sides $[F(1,7)=15.8, p<.005]$. In contrast, when the first and second stimuli differed in terms of the arrangement of their line segments, RTs to the second stimulus were higher by $67 \mathrm{msec}$ when the gap was on the same side in the two stimuli than when on opposite sides $[F(1,7)=55$, $p<.001]$.

Gaps superimposed. RTs to the first stimulus did not differ between collinear stimuli (423 msec) and parallel stimuli $(419 \mathrm{msec})(F<1)$. RTs decreased by only $10 \mathrm{msec}$ between the first and the second stimuli $(F<1)$, but this decrease was not homogeneous across conditions. The pattern of results was identical to that in all preceding blocks of this study: When the first and second stimuli were identical in terms of the arrangement of their line segments, RTs to the second stimulus were shorter by $47 \mathrm{msec}$ when the gap was on the same side in the two consecutive stimuli than when it was on opposite sides $[F(1,7)=9.2, p<$ $.05]$. In contrast, when the two stimuli differed in terms of the arrangement of their line segments, RTs to the sec- 

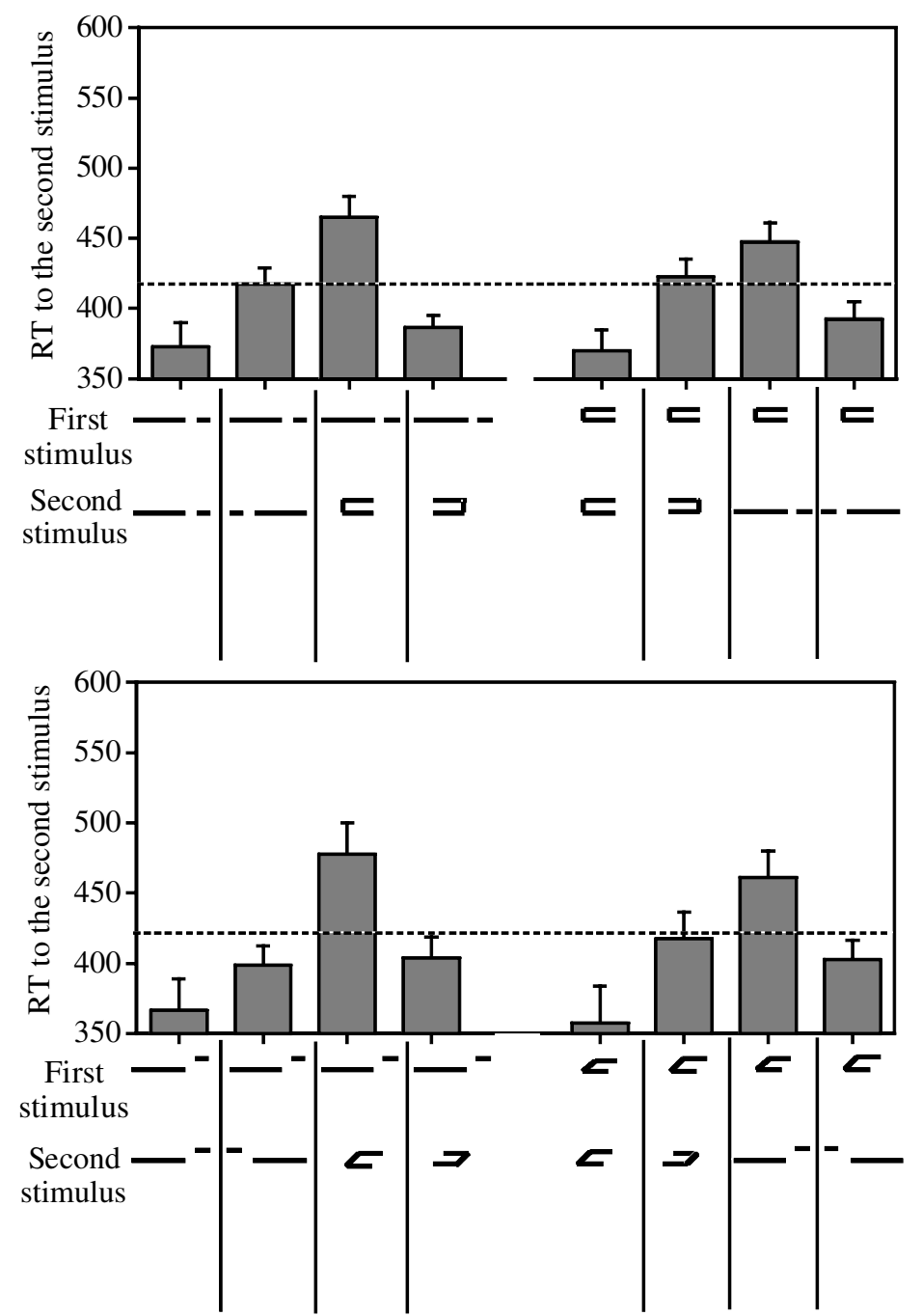

Figure 4. Mean reaction times (RTs) in Experiment 3 averaged across the 8 subjects for the second stimulus (with standard errors), as a function of experimental condition, when the stimuli are distorted and the gaps have the same orientation (lower panel) and when the stimuli are not distorted and the gaps are orthogonal (upper panel). Mean RTs for the first stimulus were homogeneous across conditions and are represented by the horizontal dotted line.

ond stimulus were higher by $66 \mathrm{msec}$ when the gap was on the same side in the first and second stimuli than when it was on opposite sides $[F(1,7)=25.2, p<.005]$. These effects resulted in a significant interaction between the relative arrangement of the two stimuli (identical vs. different), the gap location (on the same vs. on opposite sides in the two stimuli), and the time of presentation (first vs. second of a pair) $[F(1,7)=41.1, p<.001$; Figure 4 , lower panel].

Comparison between the two experimental blocks. There was no significant difference between the effects observed in the two experimental blocks.

\section{Discussion}

The manipulation of the relative orientation of the gaps in stimuli composed of collinear and parallel stimuli did not affect the results. RTs varied in the same way when gaps were orthogonal and when they were superimposed. As in Experiments 1 and 2, when the gaps in the two consecutive stimuli differed in terms of the arrangement of their line segments (composed of parallel or collinear line segments), subjects were slower when the gaps were on the same side than when they were on opposite sides. This occurred also when the two consecutive gaps were superimposed, showing that the relative orientation of the two consecutive gaps plays no role in these RT variations.

It also shows that the manipulations used in this experiment did not affect the results. Given our hypotheses concerning the modulation of early signals like virtual orthogonal lines, it might have been expected that the distortion of the parallel stimuli would have changed the results. However, it has been shown that virtual lines are 
still produced even when the virtual lines are not orthogonal with the line ends (Gillam, 1987; Gurnsey, Humphrey, \& Kapitan, 1992). Von der Heydt and Peterhans (1989) recorded cells responding to virtual lines produced by the alignment of line ends and observed that "it was the orientation of the virtual line connecting the line ends that determined the responses" (von der Heydt $\&$ Peterhans, 1989, p. 1744). Hence, the lack of impact of the distortion on the results is consistent with the literature. Concerning distorted collinear stimuli, the present results show that the collinearity of the elements plays no role in the RT variations.

\section{EXPERIMENT 4}

Experiments 2 and 3 showed that the global form of the stimuli and the relative orientation of the gaps do not explain the RT variations observed repeatedly in all experiments. If the effects observed so far really reflect modulations occurring at an early level of processing, they should be orientation and location specific. Experiment 4 was designed to test these predictions.

We tested the orientation dependency of the effect by manipulating the relative axis of the two consecutive stimuli (on the same axis in Block 1 and on orthogonal axes in Block 2). We used oblique stimuli, tilted right or left, so that changing the orientation of the stimuli did not change the task. Indeed, the use of horizontal and vertical stimuli would have forced subjects to change the type of response depending on the orientation of the stimulus (up and down vs. right and left). With oblique stimuli, in contrast, subjects can decide in all cases whether a gap is to the right or left of the center of the stimulus. If the effects are orientation sensitive, they should occur only when the two consecutive stimuli remain on the same axis (Block 1) and disappear when the two consecutive stimuli are on orthogonal axes (Block 2). Finally, we tested the location dependency of the effects by displacing the second stimulus relative to the first one (Block 3 ). Locationspecific effects should disappear when the second stimulus is displaced relative to the first one.

\section{Method}

The experiment was identical to the previous one, except that the stimuli were oriented obliquely. We took care that gaps on the same side were displayed in the exact same location on the screen. By contrast, gaps on opposite sides were never in precisely the same position on the screen. To avoid the use of a strategy based on the location of the stimulus on the screen (Figure 5), the two stimuli in a sequence were randomly displayed on the screen in a window measuring $4^{\circ}$ of visual angle horizontally and vertically. In Block 1, the two successive stimuli always remained on the same axis and in the same location, tilted right or left. In Block 2, the two stimuli were systematically oriented on orthogonal axes (one tilted rightward and one tilted leftward) and remained in the same location. In Block 3, the two successive stimuli remained on the same axis, but the second stimulus was systematically displaced relative to the first stimulus. The 8 subjects taking part in this experiment had not participated in any of the preceding experiments. The order of the experimental blocks was randomized across subjects.

\section{Results}

ANOVAs were conducted as in the preceding experiment.

RTs to the first stimulus did not differ between collinear $(533 \mathrm{msec})$ and parallel $(524 \mathrm{msec})[F(1,7)=2.1$, n.s.]. RTs decreased between the first and the second stimuli by $63 \mathrm{msec}[F(1,7)=22.2, p<.005]$. This decrease was not always homogeneous across conditions, depending on the experimental block. We will describe the results (displayed in Figure 6) for each experimental block.

Block 1: First and second stimuli on the same axis. When the two stimuli were identical in terms of the arrangement of their line segments (gaps on the same axis, Figure 6, upper panel: a, b, e, and f), RTs to the second stimulus were lower by $41 \mathrm{msec}$ when the gap was on the same side in both stimuli than when it was on opposite sides $[F(1,7)=23.9, p<.005]$. In contrast, when the two stimuli differed in terms of the arrangement of their line segments (gaps orthogonal:Figure 6, upper panel: c, d, g, and h), RTs to the second stimulus were higher by $68 \mathrm{msec}$ when the gap was on the same side in both stimuli than when it was on opposite sides $[F(1,7)=16.4, p<.005]$. These effects resulted in a significant interaction between the relative arrangement of the two stimuli (identical vs. different), gap location (on the same vs. opposite sides in the two stimuli), and the time of presentation (first vs. second of a pair) $[F(1,7)=41.5, p<.001]$.

Block 2: First and second stimuli on orthogonal axes. In Block 2, gaps in the two successive stimuli were orthogonal when the two stimuli were both made up of collinear or parallel line segments (Figure 6, middle panel: a, b, e, and $\mathrm{f}$ ). In this condition, mean RTs did not differ significantly from each other $(F<1)$. They did not differ either when the two stimuli differed in terms of the arrangement of their line segments (gaps on the same axis, Figure 6, middle panel: c, d, g, and h; $F<1$ ).

Block 3: First and second stimuli displaced. When the two stimuli were identical in terms of the arrangement of their line segments (Figure 6, lower panel: a, b, e, and f), there was an advantage of $45 \mathrm{msec}$ when the gap was on the same side in both stimuli relative to when it was on opposite sides $[F(1,7)=10.4, p<.05]$. When the two stimuli differed in terms of the arrangement of their line segments (Figure 6, lower panel: c, d, g, and h), RTs to the second stimulus were slightly higher, by $28 \mathrm{msec}$, when the gap was on the same side in both stimuli than when it was on opposite sides $[F(1,7)=4, p=.084]$. These effects resulted in a significant interaction between the relative arrangement of the line segments of the paired stimuli (identical vs. different), the side of the gap (on the same vs. opposite sides), and the time of presentation (first vs. second of a pair) $[F(1,7)=10.3, p<.05]$.

Comparison between the three experimental blocks. RTs to the first stimulus did not differ significantly in the three experimental blocks $(522,524$, and $538 \mathrm{msec}$, $F<1)$. RTs to the second stimulus were higher when the second stimulus was displaced relative to the first one (Block 3, $504 \mathrm{msec}$ ) than when the two stimuli remained 
a
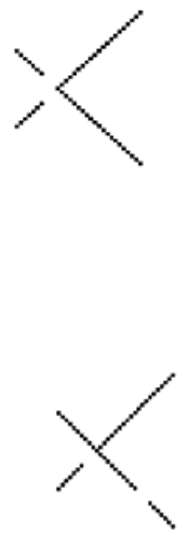

b

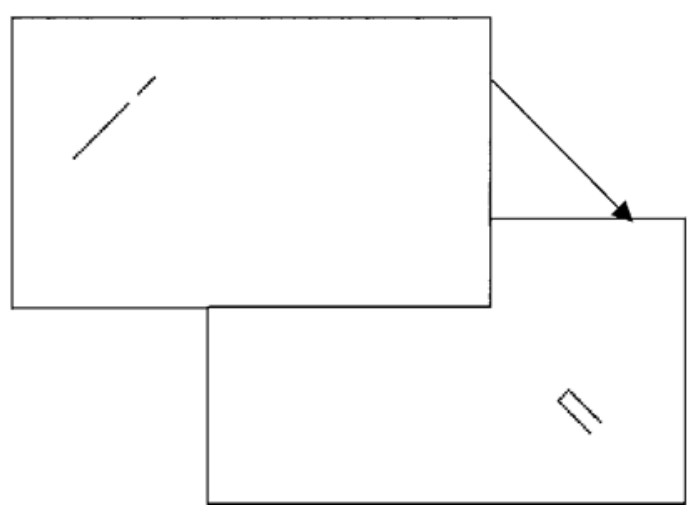

Stimuli with gaps on the same side

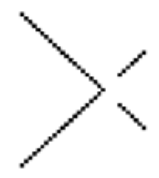

Stimuli with gaps on opposite sides
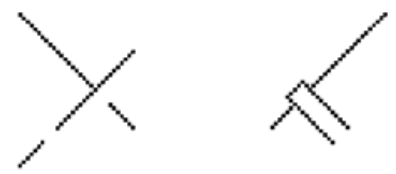
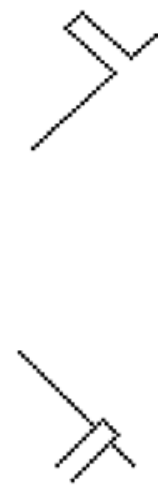

Figure 5. Stimuli are drawn superimposed (panel a) to illustrate the fact that the stimuli in Experiment 4 were presented so that the discontinuities on one side were always in the same locations on the screen in Block 2 . In Block 3 , by contrast, the second stimulus was always displaced relative to the first one (panel b).

in the same location (Blocks 1 and $2,446 \mathrm{msec})[F(1,7)=$ $28.8, p<.001]$. These effects resulted in a significant interaction between the experimental blocks and the time of presentation (first vs. second of a pair) $[F(2,14)=26.8$, $p<.001]$.

RTs increased considerably when the two consecutive stimuli were characterized by gaps on the same side and differed in terms of the arrangement of their line segments. However, this effect was significant only in Block 1, when the two successive stimuli were on the same axis and remained in the same spatial location (upper panel: $\mathrm{c}$ and $\mathrm{g}$ ). There was no significant effect anymore when the two stimuli were on orthogonal axes (Block 2), and the effect was smaller when the second stimulus was displaced relative to the first one (Block 3). As a result, when the two consecutive stimuli differed in terms of the arrangement of their line segments, the effect of gap location (on the same or on opposite sides) observed for RTs to the second stimulus differed significantly between Block 1 (stimuli on the same axis and in the same location) and Block 2 (stimuli on orthogonal axes) $[F(1,7)=24.8, p<.005]$. It also differed significantly between Block 1 (stimuli in the same location) and Block 3 (second stimulus displaced) $[F(1,7)=5.6, p<.05]$. There was no such difference between Block 2 and Block $3(F<1)$. These effects resulted in a significant interaction between the three experimental blocks, the relative arrangement of the line segments of the two stimuli (identical vs. different), gap location (on the same or opposite sides), and the time of presentation (first vs. second of a pair) $[F(2,14)=15, p<.001]$.

In Block 2 (stimuli on orthogonal axes), gaps in the two consecutive stimuli were also orthogonal when the two paired stimuli were similar in terms of the arrangement of their line segments (both composed of collinear line segments or both composed of parallel line segments). However, RTs to the second stimulus were higher by only $4 \mathrm{msec}$ when the gap was on the same side in the two consecutive stimuli (Figure 6, middle panel: a and e) relative to when they were on opposite sides (Figure 6, middle panel: $b$ and $f)$. This effect differed significantly from the 

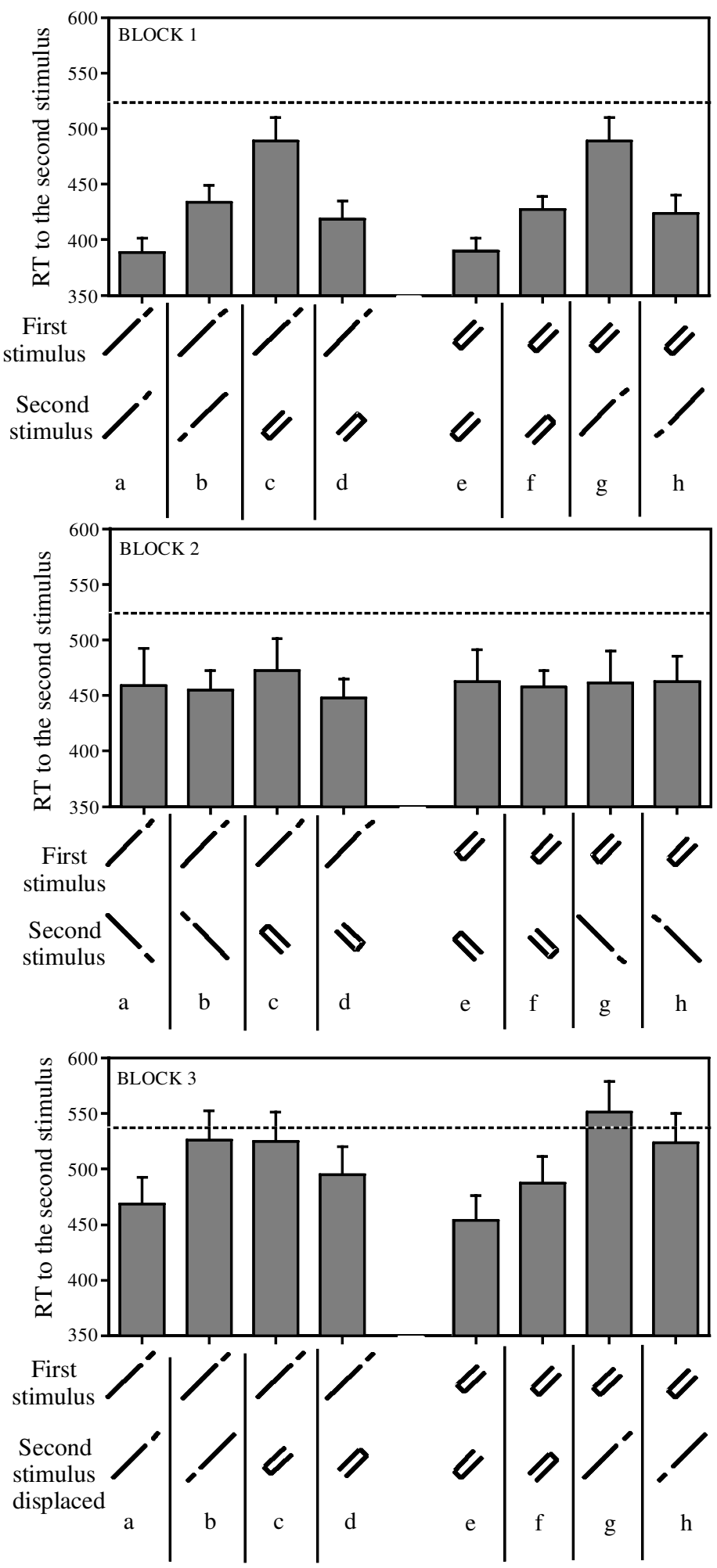

Figure 6. Mean reaction times (RTs) in Experiment 4 averaged across the 8 subjects for the second stimulus (with standard errors), as a function of experimental condition, when the axes of the first and second stimulus are identical (upper panel), when they are orthogonal (middle panel), and when the second stimulus is displaced relative to the first one (lower panel). Mean RTs for the first stimulus were homogeneous across conditions and are represented by the horizontal dotted line. 
RT increase $(68 \mathrm{msec})$ observed in Block 1 when gaps were orthogonal in two stimuli that differed in terms of the arrangement of their line segments $[F(1,7)=20, p<.005]$.

\section{Discussion}

When the two successive stimuli remained on the same axis and in the same location, subjects obviously slowed down when the gaps in the two consecutive stimuli were orthogonal and on the same side. This replicates the results of Experiment 1. However, when the two consecutive stimuli were on orthogonal axes (Block 2) or displaced relative to each other (Block 3), this RT disadvantage decreased.

In Block 2, gaps were superimposed when the two consecutive stimuli differed in terms of the arrangement of their line segments (collinear or parallel). However, the results of Experiment 3 show that this cannot account for the lack of RT variations in this block. The present results provide even more evidence that RT variations cannot be explained by the relative orientation of the two consecutive gaps. In Block 2, indeed, gaps were orthogonal when the two consecutive stimuli were both collinear or both parallel. However, in these conditions, there was no RT disadvantage when the gaps were on the same side of the two consecutive stimuli.

One might argue that the differences observed in the three blocks can be explained by a greater difficulty in Blocks 2 and 3. However, there was no difference in global performance between Blocks 1 and 2. Despite this, the effects clearly differed between these two blocks. RTs increased for the second stimulus in Block 3 . This can be easily explained, for example, by the need to find the location of the second stimulus.

As already shown in Experiment 2, the difference in the global form of the stimuli cannot explain the results. The results of Blocks 2 and 3 support this assertion further by suggesting that changing the form of the stimuli is not enough to induce RT variations. However, one might argue that the differences between Blocks 1, 2, and 3 are due to the fact that trials with two strictly identical stimuli were present only in Block 1. This may have biased subjects to alternate their responses more in Block 1 than in the other blocks, when the two consecutive stimuli differed in terms of their global form. In Block 3, there were trials with stimuli of identical form but different location. Yet, the results show that the location change did not prevent the subjects from taking advantage of the similarity of the stimuli. For these trials, the RT advantage observed when gaps were on the same side relative to when they were on opposite sides was almost identical in Block 1 (41 msec) and in Block 3 $(45 \mathrm{msec})$. This suggests that subjects did rely on the similarity of the two consecutive stimuli at least as much in Block 1 as in Block 3. Such a bias effect certainly cannot account for the whole RT variation.

The present results show that at least part of the effects are location and orientation specific and that they are not due to an antagonism between orthogonal gaps. Hence, the RT disadvantage can be attributed to the collinear versus parallel arrangement of the line segments.
However, it is important to eliminate all possible bias that may explain the results. In Experiment 5, we examined more closely the role of trials with two identical stimuli.

\section{EXPERIMENT 5}

In most of the experiments described above, a discontinuity occurred on the same side of the two consecutive stimuli in half of the trials, but these two consecutive stimuli were strictly identical in $25 \%$ of the trials and differed in $25 \%$ of the trials. The relative rarity of the trials, including two stimuli with a different form and a discontinuity on the same side, may explain the slowing down observed in this condition. This hypothesis was tested in Experiment 5 by including only trials with paired stimuli that differed in terms of the arrangement of their line segments.

\section{Method}

Experiment 5 was identical to Experiment 1 except for the elimination of all trials including two stimuli that were identical in terms of the arrangement of their line segments. The first stimulus was composed of collinear elements and the second one of parallel elements, or vice versa. In each condition, the discontinuity was either on the same side or on opposite sides in the two consecutive stimuli, defining a total of four experimental conditions (Figure 7). The experimental block included 24 trials per condition-that is, a total of 96 trials. A new group of 8 subjects participated in this experiment.

\section{Results}

RTs to the first stimulus did not differ between collinear $(457 \mathrm{msec})$ and parallel $(456 \mathrm{msec})$ stimuli $(F<1)$. RTs

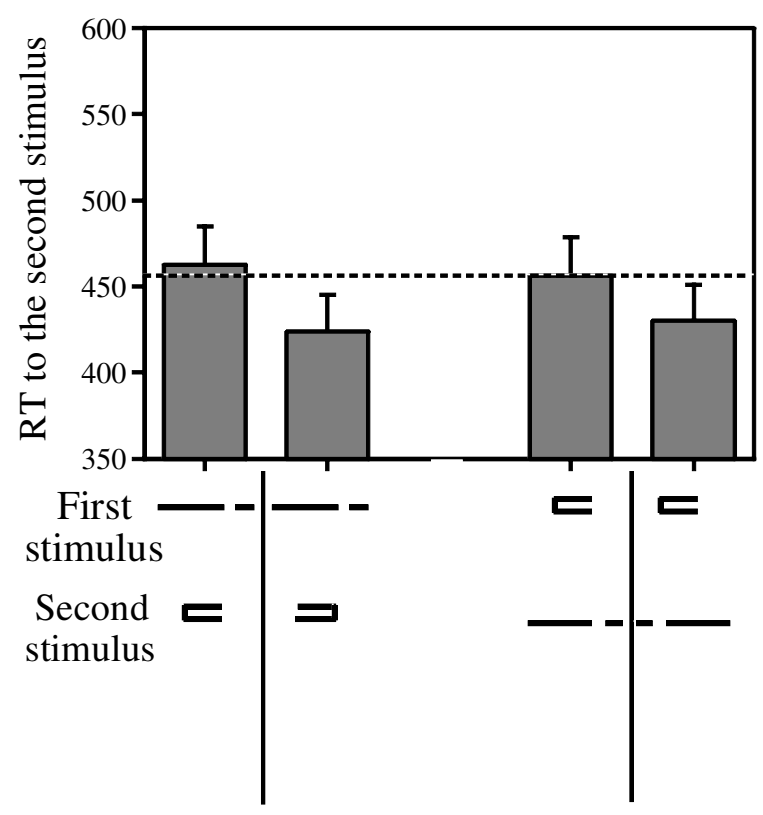

Figure 7. Mean reaction times (RTs) in Experiment 5 averaged across the 8 subjects for the second stimulus (with standard errors), as a function of experimental condition. Mean RTs for the first stimulus were homogeneous across conditions and are represented by the horizontal dotted line. 
decreased only slightly and nonsignificantly, by $13 \mathrm{msec}$, between the first and the second stimuli $(F<1)$.

RTs were higher by $32 \mathrm{msec}$ when the discontinuity was on the same side in the two consecutive stimuli than when it was on opposite sides [ $F(1,7)=15.4, p<.01$; Figure 7]. This effect resulted in a significant interaction between discontinuity location (on the same vs. opposite sides in the two stimuli) and the time of presentation (first vs. second of a pair) $[F(1,7)=9.7, p<.05]$.

\section{Discussion}

The results show that even when the two consecutive stimuli always differ in terms of the arrangement of their line segments, RTs are still higher when the gaps in both stimuli are on the same side than when they are on opposite sides. This suggests that RT variations cannot be explained only by some form of bias resulting from the presence of trials including two identical stimuli. It also confirms that the lack of effect observed in Experiment 4 can be attributed to a change in the relative orientation of the line segments or in the location of the two consecutive stimuli.

\section{EXPERIMENT 6}

In Experiment 6, we attempted to explore the hypothesis that amodal lines orthogonal to the line ends may play a role in RT variations. A frame was drawn around the stimuli composed of collinear elements. The aim of this frame was to break the orthogonal (Type 2) amodal lines that may be produced by the line ends composing the gap. If such amodal lines occur as a consequence of the presentation of a first stimulus composed of collinear elements, it may play a role in the impairment observed in the detection of a gap in the following stimulus when it is composed of parallel line segments. In that case, the breaking of this amodal line should result in less impairment. In contrast, if this amodal line is not produced or plays no role, RT variations should remain unimpaired.

However, drawing a frame around the stimulus may also result in a masking effect and thus in an impaired detection of the discontinuity. To control for this effect, additional experiments were conducted in which a frame was drawn around the stimulus composed of parallel elements. In contrast with the frame drawn around stimuli composed of collinear elements, this frame was not expected to impede the production of an amodal line orthogonal to the line ends of the parallel stimulus, since the space between the two line ends was left free. There was enough space inside the frame for an orthogonal line to impede detection of the gap. The frame was only expected to induce a similar masking effect as the frame around the stimulus composed of collinear elements. In sum, a masking effect was expected each time a frame was drawn around a stimulus, amounting to an impairment in the detection of the gap in the masked stimulus. Second, a reduction in RT variations was expected, but only when a frame was drawn around the stimulus composed of collinear elements.

\section{Method}

Subjects. Eight students attending the University of Strasbourg, the same as those in Experiment 2, took part in the tests. They had normal or corrected-to-normal visual acuity and were naive as to the aim of the study.

The procedure and apparatus were identical to those used in Experiment 1 . There were four experimental blocks. Block 1 was identical to Experiment 1. In the three remaining blocks, a frame was drawn around the stimulus composed of collinear elements (Block 2), around the stimulus composed of parallel elements (Block 3), or both (Block 4). The order of the four experimental blocks was randomized across subjects.

Stimuli. Stimuli were identical to those used in Experiment 1. Frames were rectangles drawn around the stimuli so that there was only 1 pixel $\left(2.4^{\prime}\right.$ of arc) of space between the stimulus and the frame contour (the width of the lines composing the frames was 1 pixel, $2.4^{\prime}$ of arc).

\section{Results}

RTs to the first stimulus were higher for stimuli with a frame than without any frame. When a frame was drawn only around collinear stimuli (Block 2), RTs were higher by $52 \mathrm{msec}$ for collinear stimuli than for parallel stimuli $[F(1,7)=92.8, p<.001]$. When a frame was drawn only around parallel stimuli (Block 3), RTs were higher by $41 \mathrm{msec}$ for parallel stimuli than for collinear stimuli. When there was no frame (Block 1), RTs were equivalent for collinear stimuli $(407 \mathrm{msec})$ and for parallel stimuli $(408 \mathrm{msec})(F<1)$. When frames were drawn around all stimuli (Block 4), there was a slight but significant $13 \mathrm{msec}$ disadvantage for collinear stimuli $(444 \mathrm{msec})$ relative to parallel stimuli $(431 \mathrm{msec})[F(1,7)=7.4, p<.05]$.

As in the preceding experiments, the decrease in RTs between the first and second stimuli was not homogeneous across conditions (Figure 8). However, RT variations also differed across experimental blocks, as shown by a significant interaction between experimental blocks (with vs. without a frame around the collinear stimuli-that is, Blocks 2 and 4 vs. Blocks 1 and 3), first stimulus type (with collinear or parallel line segments), gap location (on the same vs. opposite sides in the two consecutive stimuli), and the time of presentation (first vs. second of a pair) $[F(1,7)=$ $6.1, p<.05]$.

When the first stimulus was composed of collinear elements, RT variations differed significantly when there was a frame around the collinear stimulus relative to when there was no frame around the stimulus $[F(1,7)=$ $13, p<.01]$. When the two consecutive stimuli were both collinear and there was no frame drawn around them, RTs to the second stimulus were higher by $49 \mathrm{msec}$ when the gaps in the two consecutive stimuli were on opposite sides than when they were on the same side. This difference was significantly larger, by $18 \mathrm{msec}$, when a frame was drawn around the collinear stimuli $[F(1,7)=36, p<$ $.001]$. This enlargement resulted from increased RTs to the second stimulus when the gaps in the two collinear stimuli were on opposite sides: RTs were higher by $28 \mathrm{msec}$ for stimuli with a frame than for stimuli without a frame $[F(1,7)=14.6, p<.01]$. When the gaps in the two collinear stimuli were on the same side, RTs to the 

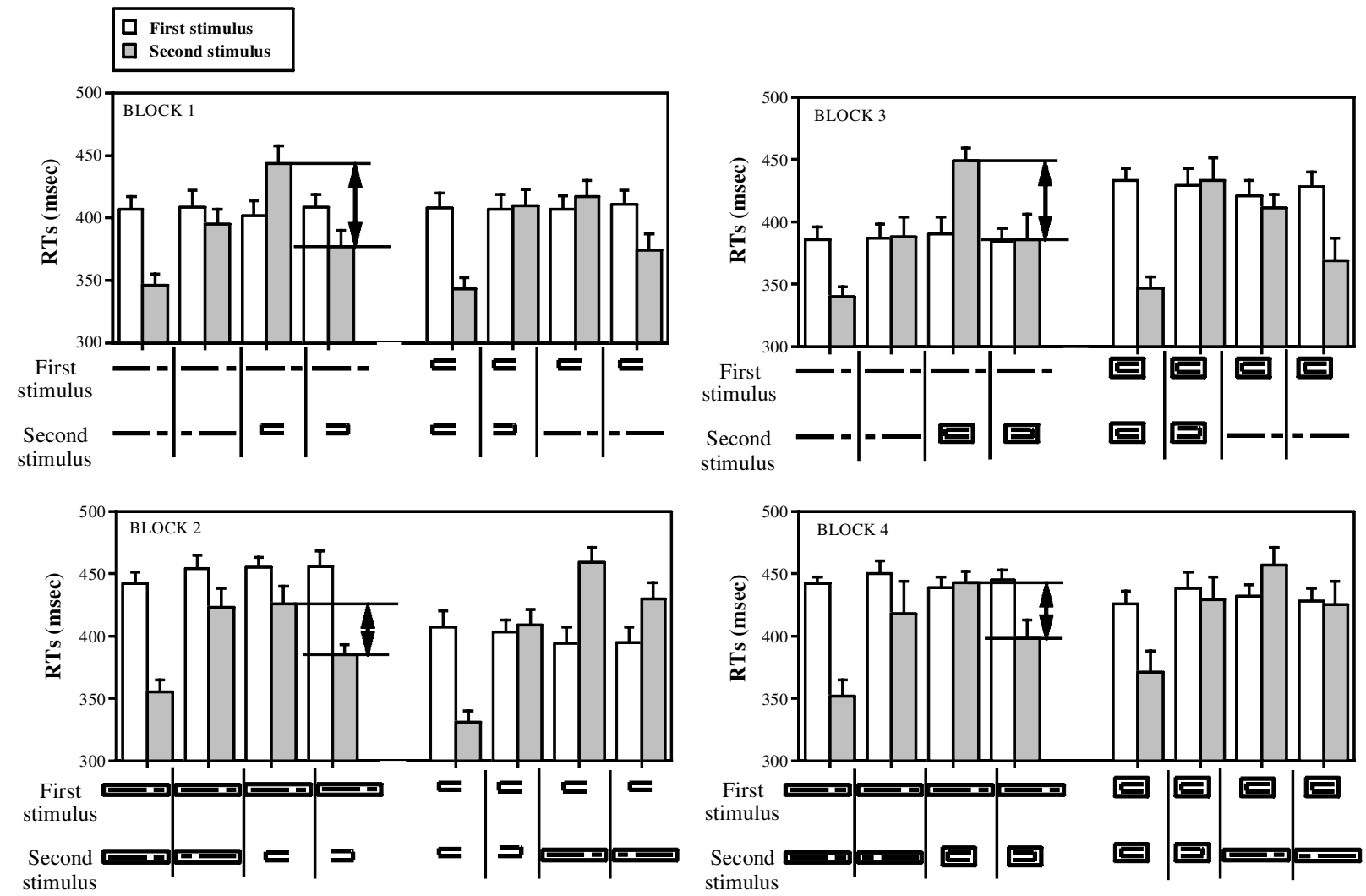

Figure 8. Mean reaction times (RTs) (with standard errors) in Experiment 6 averaged across the 8 subjects for the first stimulus (columns in white) and for the second stimulus (columns in black), as a function of experimental condition, (1) when there is no frame around the stimuli (upper left panel), (2) when there is a frame only around the "collinear" stimulus (lower left panel), (3) when there is a frame only around the "parallel" stimulus (upper right panel), and (4) when there is a frame around all stimuli (lower right panel).

second stimulus increased by only 10 msec for stimuli with a frame as relative to stimuli without a frame $(F<1)$.

When the first stimulus was collinear and the second one parallel, RTs were again higher when the gaps in two consecutive stimuli were on the same side than when they were on opposite sides. This RT disadvantage varied in amplitude according to the experimental block. When there was no frame around the collinear stimulus, there was an RT disadvantage of $66 \mathrm{msec}$ versus a disadvantage of $43 \mathrm{msec}$ when there was a frame around the collinear stimulus. These effects differed significantly $[F(1,7)=5.7, p<$ $.05]$.

In contrast, when the first stimulus was parallel, RT variations did not differ across experimental blocks $\left(F_{\mathrm{S}}<1\right)$. When both the first and the second stimuli were composed of parallel elements, there was a similar advantage (73 msec) in all experimental blocks when the gaps in the two consecutive stimuli were on the same side relative to when they were on opposite sides. When the first stimulus was parallel and the second one collinear, there was also a similar RT disadvantage $(37 \mathrm{msec})$ in all experimental blocks when the gaps of the two consecutive stimuli were on the same side relative to when they were on opposite sides.

\section{Discussion}

(1) The results showed once again that when the two consecutive stimuli were similar in terms of the arrangement of their line segments, RTs were faster when gaps were on the same side than when they were on opposite sides. This RT difference increased when stimuli were collinear and a frame was drawn around the stimuli, due to increased RTs when gaps were on opposite sides. (2) When the two consecutive stimuli differed in the arrangement of their line segments, RTs were higher when gaps were on the same side than when they were on opposite sides. This effect was also sensitive to the presence of a frame around the collinear stimulus. When a collinear stimulus was followed by a parallel stimulus with a gap on the same side, the RT disadvantage decreased when there was a frame around collinear stimuli relative to when there was no such frame. (3) The frame drawn around the parallel stimulus did not have any effect on RT variations, showing that the masking effect of the frame is not responsible for these variations.

The results are consistent with the hypothesis that the frame drawn around the collinear stimulus reduces the size of the amodal orthogonal line and affects RT variations in this way. 
First, the results show that it is slightly more difficult to detect a discontinuity between collinear than between parallel elements with a frame. A difficulty in detecting the gap between two collinear elements in the presence of a frame is also observed for responses to the second stimulus. When two collinear stimuli have a gap on opposite sides, RTs to the second stimulus are indeed higher when there is a frame around the stimuli than when there is no frame. There is no similar effect in the case of parallel stimuli. These results suggest that the frame makes it more difficult to detect a gap between collinear elements. Since we assumed that the frame would break orthogonal amodal lines in the case of collinear stimuli, these results suggest that amodal orthogonal lines do indeed play a role in the detection of a gap between collinear elements.

Second, drawing a frame around the first collinear stimulus reduces the difficulty in detecting the gap in the following parallel stimulus when the two consecutive gaps are on the same side relative to when the two stimuli have gaps on opposite sides. This supports the hypothesis that amodal orthogonal lines (Type 2) produced by the line ends in collinear stimuli may also play some role in the RT variations observed in the present study.

\section{EXPERIMENT 7}

The preceding experiments showed that the processing of a gap between two collinear line segments has an impact on the processing of a gap between two parallel line segments, provided that these gaps are in the same location and the line segments composing the two gaps share the same orientation. Experiment 7 was aimed at examining the extent to which these effects require that attention be selectively drawn to the first gap. The antagonism between stimuli composed of collinear and parallel line segments might indeed rely on mechanisms of cortical information processing occurring automatically and in parallelin other words, being applied to all the gaps appearing in the visual display. In this case, similar effects should be observed both when there is only one gap and when there are two gaps in the first stimulus. Alternatively, if the processing of discontinuities is modulated only when attention is focused on one part of an object, as we supposed in the introduction, such modulations should not be observed when there is more than one gap. In that case, attention would either be divided or drawn alternately to one gap or the other. In both cases, selective attention to a single gap would be reduced.

We examined these two possibilities by comparing RT variations when the first stimulus included only one as opposed to two gaps.

\section{Method}

Stimuli. The same stimuli were used as in Experiment 1, but with two additional stimuli. One stimulus consisted of three collinear line segments composing two gaps. The central line segment was 11 pixels long (26.4' of arc), and the two lateral line segments were each 7 pixels long (16.8' of arc). They were separated from each other by two horizontal gaps of 3 pixels each ( $7.2^{\prime}$ of arc), one on the right and the other on the left of the stimulus (Figure 9). The second additional stimulus used in this experiment was composed of two horizontal and parallel line segments, each 12 pixels long $\left(28.8^{\prime}\right.$ of arc) and separated on both sides by a vertical gap of 3 pixels $\left(7.2^{\prime}\right.$ of arc). The total length of the stimuli was identical to the length of the corresponding stimuli with only one gap.

Procedure. The procedure was similar to that in Experiment 1, with the exception that subjects were instructed not to respond to the first stimulus. The first stimulus remained on the screen for $420 \mathrm{msec}$. It was followed by a 50-msec sound, during which the screen was black. Since subjects had to withhold their responses after the first stimulus, it was important to help them to discriminate the first from the second stimulus. The sound was thus meant to announce the second stimulus, just as the first stimulus announced the second stimulus in previous experiments. The second stimulus stayed on the screen until the subjects gave their responses. The task was the same as described in previous experiments. Four experimental conditions were added in which the first stimulus included two gaps on both sides. (1) The first stimulus was composed of collinear line segments with two gaps and the second stimulus was composed of collinear line segments with only one gap, or (2) of parallel line segments with one gap. (3) The first stimulus was composed of parallel line segments with two gaps and the second stimulus was composed of parallel line segments with only one gap, or (4) of collinear line segments with only one gap.

\section{Results}

When there was only one gap in the first stimulus, the results reproduced those observed in the preceding experiments, as displayed in Figure 9 (upper panel and lower left panel). When the two consecutive stimuli were identical in terms of the arrangement of their line segments, RTs were shorter by $34 \mathrm{msec}$ when the gaps were on the same side than when they were on opposite sides $[F(1,7)=$ $10.3, p<.05]$. In contrast, when the two consecutive stimuli differed in terms of the arrangement of their line segments, RTs were higher by $46 \mathrm{msec}$ when the gaps were on the same side than when they were on opposite sides $[F(1,7)=32.9, p<.001]$. These effects resulted in a significant interaction between the relative arrangement of the stimuli (identical vs. different) and the location of the gap (on the same or opposite sides in the two consecutive stimuli) $[F(1,7)=68.3, p<.001]$.

When the first stimulus had two gaps, the variable gap location (on the same vs. opposite sides in the two stimuli) disappeared. Hence, we averaged performance over this variable when the first stimulus had only one gap, so that the effect of the relative arrangement of the two consecutive stimuli (identical vs. different) could be compared when the first stimulus had one gap versus when it had two gaps.

When the first stimulus had one gap, RTs were shorter by $34 \mathrm{msec}$ when the arrangement of the two consecutive stimuli was identical than when it was different $[F(1,7)=$ $51.1, p<.001]$. In contrast, when the first stimulus had two gaps, there was no effect of the arrangement of the two consecutive stimuli $(F<1)$. These effects resulted in a significant interaction between the number of gaps in the first stimulus (one or two) and the relative arrangement of the two consecutive stimuli (identical vs. different) $[F(1,7)=36, p<.001]$. 


\section{No response to the first stimulus}

\section{Classical Experiment}
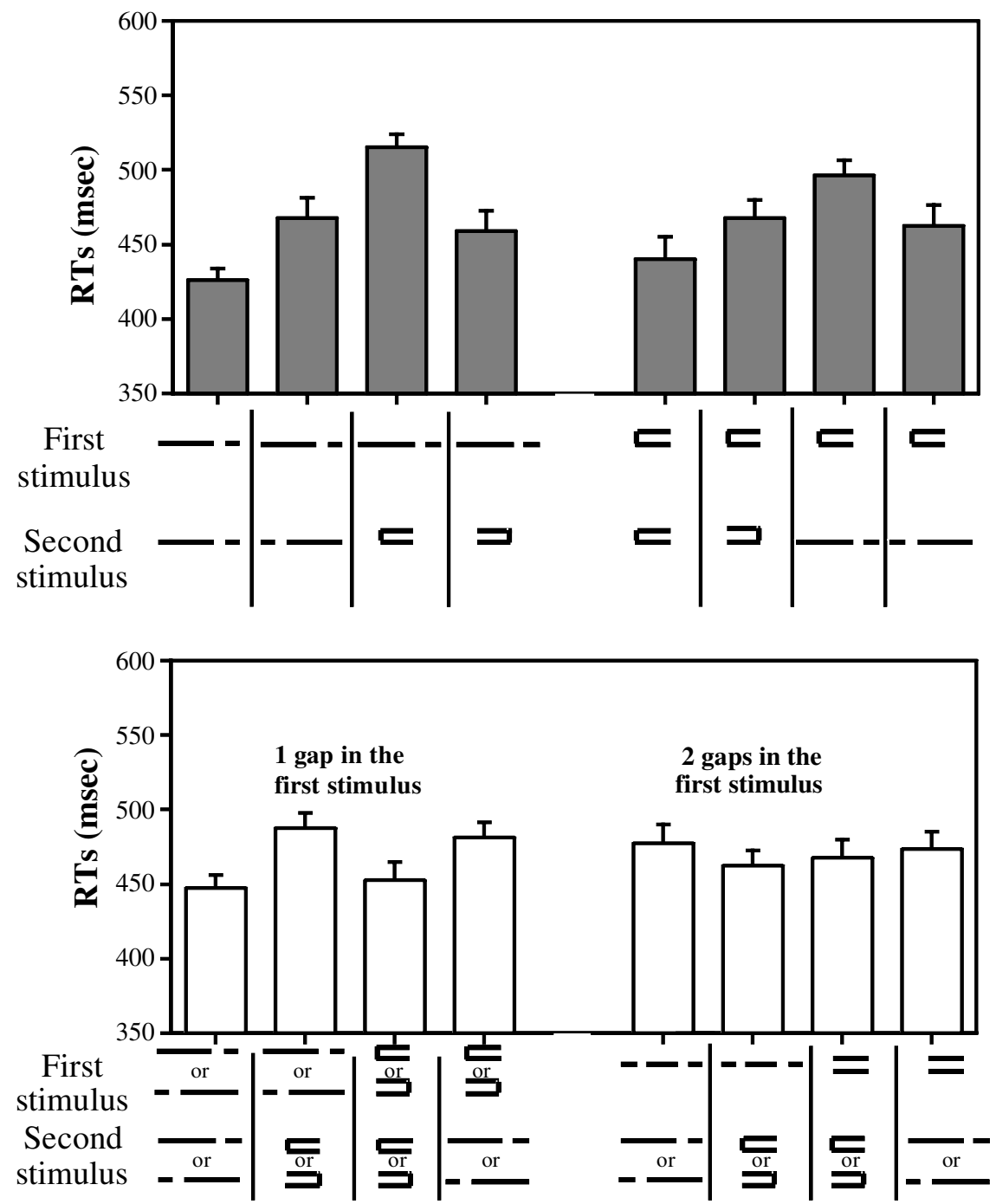

Figure 9. Mean reaction times (RTs) in Experiment 7 averaged over the 8 subjects. Subjects were instructed to give a response only after seeing the second stimulus. RTs (with standard errors) are displayed for the eight experimental conditions when the first stimulus includes one gap (upper panel). In the lower panel, RTs (with standard errors) are averaged over the locations of the gap in the second stimulus, and displayed as a function of the four remaining experimental conditions, when there is one gap in the first stimulus (left side) and when there are two gaps in the first stimulus (right side).

\section{Discussion}

The results show that, even when the subjects do not have to give a response after the first stimulus, there is still an RT disadvantage when the two consecutive stimuli differ in terms of the arrangement of their line segments and include a gap on the same side. This RT variation disappears when the first stimulus includes two gaps. These results suggest that these RT variations cannot be explained by a simple conflict between the two types of gaps or by mechanisms occurring in parallel in the visual space when- ever a gap is displayed. It probably involves more complex mechanisms such as selective attention.

\section{EXPERIMENT 8}

Experiment 7 showed that a response after the first stimulus is not required for RT variations to occur after the second stimulus. This makes it possible to examine the time course of these effects when the delay between the first and second stimulus (interstimulus interval; ISI) is ma- 
nipulated, and to examine more closely the hypothesis that RT variations are related to a forward masking effect. In a recent study, Macknik and Livingstone (1998) varied systematically ISI and target and mask durations. They confirmed that the ISI best describes forward masking. In their task, subjects had to decide which of two lines of suprathreshold brightness was the longest. Macknik and Livingstone found that maximum forward masking was observed for an ISI of $20 \mathrm{msec}$ only. However, the task used in the present experiments was different. Also, forward masking has been described up to $200 \mathrm{msec}$ of ISI (Breitmeyer, 1984). If RT variations are somehow related to a forward masking effect, these effects should diminish very quickly as the ISI increases, and disappear when ISIs are above $200 \mathrm{msec}$. In contrast, RT variations persisting for ISIs above $200 \mathrm{msec}$ would be inconsistent with an hypothesis in terms of a forward masking effect.

In addition, since these experiments required a large amount of trials, we checked the stability of the RT variations across several experimental blocks.

\section{Method}

The same stimuli were used as in Experiment 1. The procedure was identical to that described in Experiment 6, except that the first stimulus always included only one gap. The screen remained black for the whole ISI. The ISI included a first delay of $25 \mathrm{msec}$, followed by a sound during $25 \mathrm{msec}$, and then a further delay. This further delay was calculated so that the whole duration of the ISI was of 150, 300,600 , or $1,200 \mathrm{msec}$. As in the preceding experiments, there were 24 trials per experimental condition for each delay. All trials were presented in random order, whatever the ISI. Experiment 8 included six blocks of 128 trials, yielding a total of 768 trials.

\section{Results}

Effects of the ISI. There was a main effect of ISI $[F(3,21)=5.9, p<.005]$. Mean RTs, as displayed in Figure 10, were slightly higher for the ISI of $150 \mathrm{msec}$ $(480 \mathrm{msec})$ than for the other ISIs $(464,464$, and $459 \mathrm{msec}$ for the ISIs of 300, 600, and 1,200 $\mathrm{msec}$ ).

As in preceding experiments, RTs varied across experimental conditions, as suggested by a significantinteraction between the relative arrangement of the stimuli (identi-

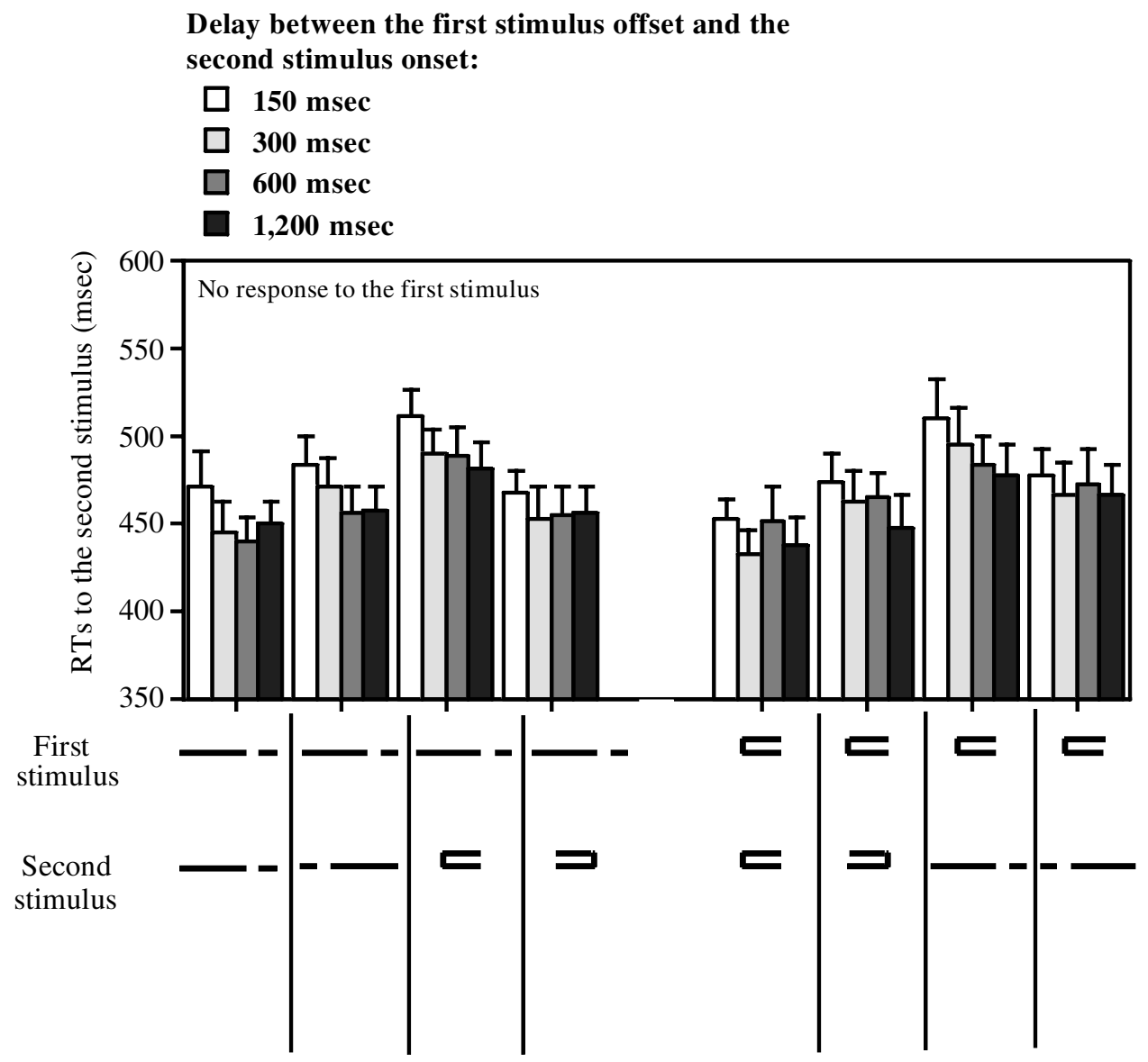

Figure 10. Mean reaction times (RTs) (with standard errors) in Experiment 8 averaged over the 8 subjects, as a function of the experimental condition and the interstimulus interval $(150,300,600$, or $1,200 \mathrm{msec}$ ). Subjects were instructed to give a response only after seeing the second stimulus. 
cal vs. different) and the location of the gap (on the same vs. opposite sides in the two consecutive stimuli) $[F(1,7)=$ $36.1, p<.001]$. However, these effects varied with the ISI, as suggested by a significant interaction between the ISI $(150,300,600$, or $1,200 \mathrm{msec})$, the relative arrangement of the stimuli (identical vs. different), and the location of the gap (on the same or on opposite sides in the two consecutive stimuli) $[F(3,21)=3.2, p<.05]$.

When the two consecutive stimuli were identical in terms of the arrangement of their line segments, RTs were shorter by $17 \mathrm{msec}$ when the gaps were on the same side than when they were on opposite sides $[F(1,7)=11.9, p<$ .05]. This advantage did not differ significantly across ISIs $(28,17,15$, and $8 \mathrm{msec}$ for the ISI of $150,300,600$, and $1,200 \mathrm{msec}$, respectively; $F \mathrm{~s}<1.7$, n.s.).

When the two consecutive stimuli differed in terms of the arrangement of their line segments, RTs were higher by $28 \mathrm{msec}$ when the gaps were on the same side than when they were on opposite sides $[F(1,7)=43.6, p<.001]$. This effect decreased linearly as the ISI increased $(39,33,23$, and $18 \mathrm{msec}$ for the ISI of $150,300,600$, and $1,200 \mathrm{msec}$ respectively), as suggested by a significant interaction between the ISI and the location of the gap (on the same vs. opposite sides), observed in the polynomial test of order 1 $[F(1,7)=11.8, p<.05]$. An effect of the location of the gap was largely significant for the three shortest ISIs $(F \mathrm{~s}>$ $21.4, p \mathrm{~s}<.005)$, but tended to be significant only for the ISI of $1,200 \mathrm{msec}[F(1,7)=4.7, p=.067]$.

Effects of training. The effects of training were examined by pooling RTs across all ISIs (similar results were observed when only the two or three shortest ISIs were taken into consideration). The whole experiment, including six blocks, was divided into three sets of data. Each set included the results of two consecutive blocks, or 32 trials per condition.

There was a main effect of data set $[F(2,14)=6.3, p<$ $.05]$. RTs decreased significantly between the first data set $(487 \mathrm{msec})$ and the second one $(460 \mathrm{msec})[F(1,7)=$ $5.9, p<.05]$ and remained stable between the second and third data sets $(454 \mathrm{msec})[F(1,7)=1.9$, n.s.].

Data sets did not interact significantly with the other variables in the main analysis. However, a closer examination of the results was conducted to check the impact of training on RT variations. When the two consecutive stimuli were identical in terms of the arrangement of their line segments, the RT advantage for gaps on the same side relative to gaps on opposite sides varied significantly in amplitude across data sets $[F(2,14)=5.3, p<.05]$. In the first set of data, RTs were only slightly $(9 \mathrm{msec})$ and nonsignificantly shorter when gaps were on the same side than when they were on opposite sides $[F(1,7)=1.8$, n.s.]. This advantage increased and became significant only by the second set of data [16 msec, $F(1,7)=10.2, p<.05]$ and the third one [22 msec, $F(1,7)=30, p<.001]$.

When the two consecutive stimuli differed in terms of the arrangement of their line segments, data set did not interact with any other variable $\left(F_{\mathrm{s}}<1\right)$. RTs were systematically higher when gaps were on the same side than when they were on opposite sides, by $28 \mathrm{msec}$ in the first data set $[F(1,7)=13.2, p<.01], 30 \mathrm{msec}$ in the second data set $[F(1,7)=69.6, p<.001]$, and $23 \mathrm{msec}$ in the third $[F(1,7)=18.1, p<.005]$.

\section{Discussion}

The results show that RT variations decline slowly as the time interval between the first and second stimuli increases. Significant RT variations are still observed for an ISI of $600 \mathrm{msec}$, and these effects only partially disappear for the interval of $1,200 \mathrm{msec}$ (stimulus onset asynchronies [SOAs] are always $420 \mathrm{msec}$ longer). Such a long-lasting effect is not compatible with an explanation in terms of a forward masking effect (Breitmeyer, 1984).

The stability of RT variations across experimental blocks also confirms the robustness of these effects. When the two consecutive stimuli were identical, the RT advantage for trials with two gaps on the same side relative to trials with gaps on opposite sides tended to increase across experimental blocks. These results suggest that subjects required some time to take advantage of the similarity between the first and second stimuli. In contrast, when the two consecutive stimuli differed in terms of the arrangement of their line segments, the RT disadvantage for trials including gaps on the same side relative to trials including gaps on opposite sides remained stable. This confirms the results of Experiment 5, showing that trials including two identical stimuli do not account for RT variations in trials including two different stimuli.

\section{GENERAL DISCUSSION}

The main results can be summarized as follows: (1) RTs for a given stimulus vary as a function of the preceding stimulus type. When the first and second stimuli share the same line-segment arrangement (either collinear or parallel), RTs are shorter when the gap is on the same side in both stimuli than when it is on opposite sides. In contrast, when the first and second stimuli differ in terms of the arrangement of their line segments, RTs are higher when the gap is on the same side than when it is on opposite sides. (2) This RT increase is reproduced in seven groups of 8 subjects each. (3) Experiment 2 shows that this effect cannot be explained by a difference in the global forms of the two consecutive stimuli. It is observed even if the global forms of the stimuli are very similar. (4) RT variations are not due to the orthogonality of the two consecutive gaps either. They are still observed when the two consecutive gaps are superimposed (Experiment 3). (5) Experiment 4 shows that RT variations are observed only when the line ends forming the gap in the first and second stimuli share the same location and orientation. They disappear when the orientation of the line ends forming the gap differs by $90^{\circ}$ between the first and the second stimuli, and decrease significantly when the second stimulus is displaced relative to the first one. (4) These results are not due to the presence of trials including two identical stimuli (Experiment 5). (5) Drawing a frame around the 
collinear stimulus also affects RT variations. When the first stimulus is collinear and the second one parallel with a gap on the same side, the RT disadvantage decreases when there is a frame around the collinear stimulus (Experiment 6). (6) There is still an RT disadvantage when subjects do not give a response to the first stimulus, provided the first stimulus includes only one gap. RTs do not vary when the first stimulus includes two gaps (Experiment 7). (7) RT variations persist for delays as long as $600 \mathrm{msec}$ between the first and second stimuli (Experiment 8). (8) RT variations persist across several experimental blocks (Experiment 8).

These results show that the detection of a gap is influenced by the nature of the gap displayed immediately before. They suggest that the collinear versus parallel arrangement of the two consecutive stimuli plays a critical role in the RT increase observed when the gap remains on the same side in the two stimuli. Indeed, the fact that, in some conditions, RTs to the second stimulus increase when the gap remains on the same side shows that there is more than a simple activation of the side of the gap. The manipulation of the similarity of the stimuli (Experiment 2), the orientation and location specificity of the effect (Experiment 4), and the persistence of RT variations in the absence of trials including two strictly identical stimuli (Experiment 5) all suggest that this influence is not due to a nonspecific bias effect. However, it might still be argued that expectations or a strategy based on the display of the first stimulus could have some orientation and location-specific consequences on the processing of the second stimulus. This would mean that a gap between two collinear elements would be in some way incompatible with a gap between two parallel elements in the same location and with the same orientation. However, subjects were not aware of any incompatibility between the two types of gaps and were rather surprised when they saw their results. In particular, they never reported interpreting the stimuli as being occluded or as including an amodally completed gap. Moreover, given the considerable ambiguity of the stimuli, RT variations relying on set and expectancies should have been more variable across subjects, which was not the case. On the contrary, the effects were very robust. In particular, strategies should have been sensitive to the repetition of experimental blocks. With training, subjects may have changed their strategy, especially since there was no obvious incompatibility between the two types of gaps. Experiment 8 shows that, on the contrary, RT variations were resistant to training. Finally, such strategies should have been insensitive to the addition of a frame around the stimuli, since the configuration of the gaps remained identical. This was not the case, as shown by Experiment 6 . For all these reasons, an explanation in terms of decision-making mechanisms is not adopted here, even if this interpretation is necessarily limited by the lack of direct proof concerning the level at which modulations really occur.

Several low-level mechanisms could account for the results. The experiments make it possible to exclude some of these. The manipulation of the physical stimulus properties showed that orthogonal gaps in the two consecutive stimuli are not enough to produce the effect (Experiment 4) and that the effect is still observed when the gaps are superimposed (Experiment 3 ). A classical forward masking effect also seems unlikely. Given that the configuration of the gaps plays a critical role in RT variations, a forward masking effect would imply that a gap between collinear line segments and a gap between parallel line segments mask each other. In this case, RT variations should have been preserved in Experiment 6, when a frame was drawn around the stimuli because the configuration of the gaps remained intact. That was not the case. In Experiment 7, too, the gaps were composed of collinear or parallel elements, even when there were two gaps. A masking effect should have been preserved in Experiment 7, which was not the case. Finally, an account in terms of a classical forward masking effect is not compatible with the persistence of the RT variations above $200 \mathrm{msec}$ of ISI (Breitmeyer, 1984).

If the collinear and parallel arrangement plays a role in the RT variations observed after the display of the second stimulus, not only one but several mechanisms might underlie these variations. The results show clearly that not all types of discontinuities are equivalent. It seems also that modulations in the processing of discontinuities occur, depending on physical properties like the arrangement of line segments. Though the following hypotheses should be viewed with caution, it seems to us that the present results may help to discriminate between several mechanisms underlying modulations in the processing of discontinuities.

A first possibility is that gaps that can be completed with Type 1 amodal contours, such as gaps between collinear elements, are incompatible with open ends, just like gaps between parallel elements, which cannot be completed with Type 1 contours. However, the mechanisms of this incompatibility should still be explained: To our knowledge, there is no argument for the existence of a reciprocal inhibition between the two types of gaps. Moreover, this incompatibility should have persisted when a frame was drawn around the stimulus (Experiment 6), since the configuration of the gap remained the same. That was not the case.

When the task is to detect a gap between collinear line segments, it might be useful (1) to inhibit the amodal line linking the two line ends, (2) to activate the production of amodal lines orthogonal to the line ends, or (3) to activate the processing of the line ends, which indicate the presence of a gap. It is not clear why inhibiting the amodal line linking the two line ends should have a deleterious effect on the detection of a gap between parallel line segments. On the other hand, amodal lines orthogonal to the line ends might help to detect the gap in collinear stimuli, for example, because these orthogonal lines are cues to line ends and cannot impair the detection of the gap (they are not superimposed on the gap, as is the case for stimuli composed of parallel line segments). In particular, they might help to reconcile a tendency toward an amodal com- 
pletion between the two collinear line segments and the task requiring the detection of the gap, by playing the role of an occluder in the foreground. The hypothesis that amodal lines orthogonal to the line ends play a role in the results is supported by the results of Experiment 6. Indeed, these results suggest that breaking these amodal orthogonal lines by means of a frame drawn around the collinear stimulus impairs the detection of the gap and affects RT variations. By contrast, in the case of parallel stimuli, the production of a line orthogonal to the line ends leads to an ambiguity concerning the location of the gap because it implies the presence of a contour at the location of the gap. It follows that the gaps in collinear stimuli are compatible with the presence of an occluder at the location of the gap, whereas gaps in parallel stimuli are not. As noted, subjects never reported interpreting the stimuli as being occluded. It thus seems unlikely that RT variations were due to an incompatibility between two types of interpretations. It is more likely that the effects rely on a low-level activation of amodal lines or line ends. One possibility might be that amodal lines orthogonal to the line ends are activated when the task is to detect a gap between two collinear line segments, but need to be inhibited when the task is to detect a vertical gap between two parallel line segments. This hypothesis could explain a reciprocal antagonism. It is certainly not inconsistent with the similarity of the performance observed when the first stimuli are composed of collinear or parallel elements. If modulations occur, they occur for both stimuli. Also, the simplicity of the task may allow a response to the first stimulus before the modulations are achieved. These modulations may affect only the processing of the second stimulus. Several studies suggest that amodal completion develops relatively slowly over time, at least when there is no depth information (Bruno, Bertamini, \& Domini, 1997; Sekuler, 1994; Sekuler \& Palmer, 1992). The present results are also compatible with studies suggesting that occluded contours can be completed at an early stage of processing, even if they do not have the same status as real lines (Giersch et al., 2000; Lesher, 1995; Rensink \& Enns, 1998). In accordance with these studies, our results suggest that the production of amodal lines, either linking the line ends or orthogonal to the line ends, may occur without reaching consciousness but nevertheless influence the processing of discontinuities.

Another possibility is that RT variations are based on the activation of the processing of line ends. Such an activation may help to detect the gap between two collinear elements. In the case of parallel line segments, however, the alignment of line ends leads to the production of orthogonal amodal lines (Type 2). It follows that the activation of the processing of line ends might increase the strength of these amodal lines and impair the detection of a gap between parallel line segments.

Since end-stopped cells have been proposed as the physiological substrate for the coding of line ends (Dobbins, Zucker, \& Cynader, 1987; Hubel \& Wiesel, 1965; Saito, Tanaka, Fukada, \& Oyamada, 1988; van der Zwan, Baumann, \& Peterhans, 1995), it might be tempting to propose that the modulation of the processing of line ends reflects a modulation of end-stopped cell responses. The results are consistent with what is known about the properties of endstopped cells, in particular their orientation and location specificity, and suggest that the responses of these cells are more plastic than once believed. Modulations of the responses of end-stopped cells have already been proposed by Yu and Levi (1999). These authors measured the contrast threshold for detecting a masked target. When the mask was long enough to encroach on end zones, the masking effect decreased, revealing "psychophysical end-stopping." Yu and Levi showed that the reduction of the masking effect was observed only when the mask onset and the target onset were separated by a time interval of at least $70 \mathrm{msec}$ and developed fully up to $150-200 \mathrm{msec}$. These results suggest that psychophysical end-stopping develops relatively slowly over time.

However, the results of Experiment 7 suggest that the RT variations observed in the present study are not the result of a simple property of the cells coding the discontinuities or of an antagonism between different types of processing. Instead, they suggest that modulations in the processing of discontinuities occur only when the discontinuity is processed under selective attention conditions. It remains to be determined whether the display of a gap captures attention in a stimulus-driven fashion, as proposed for abrupt visual onsets (Egeth \& Yantis, 1997; Jonides \& Yantis, 1988; Yantis \& Jonides, 1990) or if attention has to be intentionally directed toward the gap for modulations of the processing of the gap to occur. This is important because control by goal-directed attention would imply a top-down control on early mechanisms, whereas control by stimulus-driven attention would not have the same significance. This question requires further investigation. Indeed, the present results do not make it possible to distinguish between the two hypotheses: Even when subjects do not have to respond to the first stimulus, they may nevertheless voluntarily process the information conveyed by the gap.

Whatever the precise mechanisms of the performance modulations described above, our results suggest that the detection of discontinuitiescan be modulated, probably on a low level of visual processing, in keeping with studies suggesting that the coding of primitives is more plastic than once believed (Fahle, 1996; Yeshurun \& Carrasco, 2000). The quickly evolving modulations observed in the present study might be used to focus on details, after a more global survey has been achieved, as was proposed for the processing of visual scenes (Rayner \& Pollatsek, 1992). Indeed, an initial raw processing of the available visual information, in particular of low spatial frequencies, might allow a fast categorization of objects, sufficient for a general survey of a visual scene. Thorpe, Fize, and Marlot (1996) showed, for example, that complex images can be categorized successfully within $150 \mathrm{msec}$. The activation of the processing of discontinuities might therefore help to pick out more clearly the local features of an object or a scene and to distinguish between intrinsic and extrin- 
sic line ends. Such a hypothesis does not imply that the coding of discontinuities is inactive when attention is not focused on an object part. The observation that there is no advantage for collinear or parallel stimuli when they are presented as first stimuli suggests that the visual system may be in a balanced state allowing different types of discontinuities to be detected equally well. Analysis of the precise nature of the discontinuities would be processed only if needed. For example, the signals coding amodal lines and line ends, once produced in an automatic way, may be modulated according to the needs of the task. A selective modulation of this processing might allow a more economical processing of visual information, in particular when visual scenes include many nonsignificant details. The possible role of attentional processes in the activation of discontinuities processing still has to be explored.

\section{REFERENCES}

Biederman, I. (1987). Recognition-by-components: A theory of human image understanding. Psychological Review, 94, 115-147.

Breitmeyer, B. G. (1984). Visual masking. New York: Oxford University Press.

Bringuier, V., Chavane, F., Glaeser, L., \& Frégnac, Y. (1999). Horizontal propagation of visual activity in the synaptic integration field of area 17 neurons. Science, 283, 695-699.

Bruno, N., Bertamini, M., \& Domini, F. (1997). Amodal completion of partly occluded surfaces. Is there a mosaic stage? Journal of Experimental Psychology: Human Perception \& Performance, 23, 1412-1426.

Chey, J., Grossberg, S., \& Mingolla, E. (1997). Neural dynamics of motion grouping: From aperture ambiguity to object speed and direction. Journal of the Optical Society of America A, 10, 2570-2594.

DeAngelis, G. C., Freeman, R. D., \& Ohzawa, I. (1994). Length and width tuning of neurons in the cat's primary visual cortex. Journal of Neurophysiology, 71, 347-374.

Dobbins, A., Zucker, S. W., \& Cynader, M. S. (1987). Endstopped neurons in the visual cortex as a substrate for calculating curvature. Nature, 329, 438-441.

Duysens, J., Orban, G.A., van der Glas, H.W., \& de Zegher, F. E. (1982). Functional properties of area 19 as compared to area 17 of the cat. Brain Research, 231, 279-291.

Duysens, J., Orban, G. A., van der Glas, H. W., \& Maes, H. (1982). Receptive field structure of area 19 as compared to area 17 of the cat. Brain Research, 231, 293-308.

Egeth, H. E., \& YANTIS, S. (1997). Visual attention: Control, representation and time course. Annual Review of Psychology, 48, 269-297.

Eysel, U. T., Eyding, D., \& Schweigert, G. (1998). Repetitive optical stimulation elicits fast receptive field changes in mature visual cortex. NeuroReport, 9, 949-954.

FAHLE, M. (1994). Human pattern recognition: Parallel processing and perceptual learning. Perception, 23, 411-427.

FAHLE, M. (1996). Human object recognition: Parallel processing and perceptual learning. Recent Research Developments in Biological Cybernetics, 1, 91-105.

Giersch, A. (1999). A new pharmacological tool to investigate integration processes. Visual Cognition, 6, 267-297.

Giersch, A., Humphreys, G., Boucart, M., \& Kovacs, I. (2000). The computation of contours in visual agnosia: Evidence for early computation prior to shape binding and figure-ground coding. Cognitive Neuropsychology, 17, 731-759.

Gilbert, C. D., Das, A., Ito, M., Kapadia, M., \& Westheimer, G. (1996). Spatial integration and cortical dynamics. Proceedings of the National Academy of Sciences, 93, 615-622.

Gillam, B. (1987). Perceptual grouping and subjective contours. In S. Petry \& G. E. Meyer (Eds.), The perception of illusory contours (pp. 268-273). New York: Springer-Verlag.
Grosof, D. H., Shapley, R. M., \& Hawken, M. J. (1993). Macaque V1 neurons can signal "illusory" contours. Nature, 365, 550-552.

Grossberg, S., \& Mingolla, E. (1985). Neural dynamics of perceptual grouping: Textures, boundaries, and emergent segmentations. Perception \& Psychophysics, 38, 141-171.

Gurnsey, R., Humphrey, G. K., \& Kapitan, P. (1992). Parallel discrimination of subjective contours by offset gratings. Perception \& Psychophysics, 52, 263-276.

Gurnsey, R., Iordanova, M., \& Grinberg, D. (1999). Detection and discrimination of subjective contours defined by offset gratings. Perception \& Psychophysics, 61, 1256-1268.

Heitger, F., Rosenthaler, L., von der Heydt, R., Peterhans, E., \& Kubler, O. (1992). Simulation of neural contour mechanisms: From simple to end-stopped cells. Vision Research, 32, 963-981.

Hubel, D. H., \& WiESEL, T. N. (1965). Receptive fields and functional architecture in two nonstriate visual areas (18 and 19) of the cat. Journal of Neurophysiology, 28, 229-289.

Humphreys, G. W., \& RidDOch, J. (1994). Attention to within-object and between-object spatial representations: Multiple sites for visual selection. Cognitive Neuropsychology, 11, 207-241.

Hupé, J. M., James, A. C., Payne, B. R., Lomber, S. G., Girard, P., \& BULliER, J. (1998). Cortical feedback improves discrimination between Figure and background by V1, V2 and V3 neurons. Nature, 394, 784787.

JoNidES, J., \& YANTIS, S. (1988). Uniqueness of abrupt visual onset in capturing attention. Perception \& Psychophysics, 43, 346-354.

KANIZSA, G. (1979). Organization in vision: Essays on Gestalt perception. New York: Praeger.

Kapadia, M. K., Ito, M., Gilbert, C. D., \& Westheimer, G. (1995). Improvement in visual sensitivity by changes in local context: Parallel studies in human observers and in V1 of alert monkeys. Neuron, 15, 843-856.

Kellman, P. J., \& Shipley, T. F. (1991). A theory of visual interpolation in object perception. Cognitive Psychology, 23, 141-221.

KenNedy, H., \& Bullier, J. (1985). A double-labelling investigation of the afferent connectivity to cortical areas V1 and V2 of the macaque monkey. Journal of Neuroscience, 5, 2815-2830.

LESHER, G. W. (1995). Illusory contours: Toward a neurally based perceptual theory. Psychonomic Bulletin \& Review, 2, 279-321.

Lesher, G. W., \& Mingolla, E. (1993). The role of edges and lineends in illusory contour formation. Vision Research, 33, 2253-2270.

LorenceaU, J., \& ShIfFrar, M. (1992). The influence of terminators of motion integration across space. Vision Research, 32, 263-273.

MackniK, S. L., \& Livingstone, M. S. (1998). Neuronal correlates of visibility and invisibility in the primate visual system. Nature Neuroscience, 1, 144-149.

McKee, S. P., \& Westheimer, G. (1978). Improvement in vernier acuity with practice. Perception \& Psychophysics, 24, 258-262.

McLean, J., \& Palmer, L. A. (1998). Plasticity of neuronal response properties in adult cat striate cortex. Visual Neuroscience, 15, 177-196.

Nakayama, K., Shimojo, S., \& Silverman, G. H. (1989). Stereoscopic depth: Its relation to image segmentation, grouping, and the recognition of occluded objects. Perception, 18, 55-68.

Orban, G., Kato, H., \& Bishop, P. O. (1979a). Dimensions and properties of end-zone inhibitory areas in receptive fields of hypercomplex cells in cat striate cortex. Journal of Neurophysiology, 42, 833-849.

Orban, G., Kato, H., \& Bishop, P.O. (1979b). End-zone region in receptive field of hypercomplex and other striate neurons in the cat. Journal of Neurophysiology, 42, 818-832.

Peterhans, E., \& VON Der Heydt, R. (1991). Elements of form perception in monkey prestriate cortex. In A. Goréa, Y. Frégnac, Z. Kapoula, \& J. Findlay (Eds.), Representations of vision: Trends and tacit assumption in vision research (pp. 111-124). Cambridge: Cambridge University Press.

Poggio, T., Fahle, M., \& Edelman, S. (1992). Fast perceptual learning in visual hyperacuity. Science, 256, 1018-1021.

Rayner, K., \& Pollatsek, A. (1992). Eye movements and scene perception. Canadian Journal of Psychology, 46, 342-376.

Redies, C., Crook, J. M., \& Creutzfeldt, O. D. (1986). Neuronal reponses to borders with and without luminance gradients in cat visual 
cortex and dorsal geniculate nucleus. Experimental Brain Research, 61, 469-481.

RENSINK, R. A., \& ENNS, J. T. (1998). Early completion of occluded objects. Vision Research, 38, 2489-2505.

Saito, H., Tanaka, K., Fukada, Y., \& Oyamada, H. (1988). Analysis of discontinuity in visual contours in area 19 of the cat. Journal of Neuroscience, 8, 1131-1143.

Salin, P. A., \& Bullier, J. (1995). Corticocortical connections in the visual system: Structure and function. Physiological Review, 75, 107-154.

SEKuler, A. B. (1994). Local and global minima in visual completion: Effects of symmetry and orientation. Perception, 23, 529-545.

Sekuler, A. B., \& Palmer, S. E. (1992). Perception of partly occluded objects: A microgenetic analysis. Journal of Experimental Psychology: Human Perception \& Performance, 121, 95-111.

Sheth, B. R., Sharma, J., Rao, C., \& Sur, M. (1996). Orientation maps of subjective contours in visual cortex. Science, 274, 2110-2115.

Shiffrar, M., \& LORENCEAU, J. (1996). Improved motion linking across edges at decreased luminance contrast, edge width and duration. Vision Research, 36, 2061-2069.

Shimojo, S., \& Nakayama, K. (1990). Amodal representation of occluded surfaces: Role of invisible stimuli in apparent motion correspondence. Perception, 19, 285-299.

Shimojo, S., Silverman, G. H., \& Nakayama, K. (1989). Occlusion and the solution to the aperture problem for motion. Vision Research, 29, 619-626.

Shipley, T. F., \& Kellman P. J. (1990). The role of discontinuities in the perception of subjective figures. Perception \& Psychophysics, 48 , 259-270.

ShIPP, S., \& ZeKI, S. (1989). The organization of connections between areas V5 and V2 in macaque monkey visual cortex. European Journal of Neuroscience, 1, 333-354.

Thorpe, S., Fize, D., \& Marlot, C. (1996). Speed of processing in the human visual system. Nature, 381, 520-522. van der Zwan, R., Baumann, R., \& Peterhans, E. (1995). Endstopped cells in the visual cortex of the alert monkey. Perception, 24 (Suppl.), 43b.

von der Heydt, R, \& Peterhans, E. (1989). Mechanisms of contour perception in monkey visual cortex 1 : Lines of pattern discontinuities. Journal of Neuroscience, 9, 1731-1748.

Westheimer, G., \& LI, W. (1996). Classifying illusory contours by means of orientation discrimination. Journal of Neurophysiology, 75, 523-528.

YANTIS, S., \& Jonides, J. (1990). Abrupt visual onsets and selective attention: Voluntary versus automatic allocation. Journal of Experimental Psychology: Human Perception \& Performance, 16, 121-134.

Yeshurun, Y., \& CARrasco, M. (2000). The locus of attentional effects in texture segmentation. Nature, 3, 622-627.

Yu, C., \& Levi, D. M. (1999). The time course of psychophysical endstopping. Vision Research, 39, 2063-2073.

ZucKer, S. W., \& DAvis, S. (1988). Points and endpoints: A size/spacing constraint for dot grouping. Perception, 17, 229-247.

\section{NOTE}

1. We will call such orthogonal lines "amodal" in the remaining text, for the sake of simplicity. It should be noted, however, that this term is used in a broader sense than Kanizsa's (1979) original definition. Amodal contours are thought to give rise to a perceived completion in the absence of sensory aspects, such as brightness or color. In our studies, however, subjects were not even aware of the possibility that some orthogonal line may have been produced at the ends of the lines. For this reason, we put amodal in quotation marks.

(Manuscript received February 1, 2000; revision accepted for publication March 20, 2001.) 